Final Report

\title{
IMPROVED DETERMINISTIC CALCULATIONAL METHODS FOR IRREGULARLY SHAPED SHIELDS
}

\author{
September 30, 1988 - November 30, 1990 \\ DOE/ER/12818--2 \\ DE93 004955
}

Submitted to:

Dr. Joseph H. Nadler

U. S. Department of Energy

Idaho Field Office

785 DOE Place, MS-1220

Idaho Falls, ID 83401-1562

Submitted by:

John J. Dorning

Whitney Stone Professor

Nuclear Reactor Facility

Department of Mechanical, Aerospace and Nuclear Engineering

UNIVERSITY OF VIRGINIA SCHOOL OF ENGINEERING AND APPLIED SCIENCE

THORNTON HALL

CHARLOTTESVILLE, VA 22903-2442

SEAS Report No. UVA/527390/MANE93/102

December 1992

Copy No. 2 


\section{TABLE OF CONTENTS}

EXECUTIVE SUMMARY $\ldots \ldots \ldots \ldots \ldots \ldots \ldots \ldots \ldots \ldots \ldots$

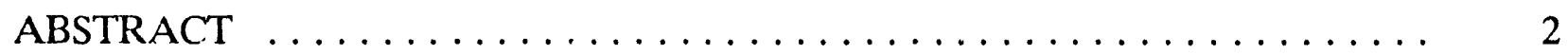

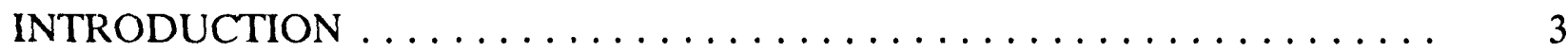

THE BOUNDARY-FITTED COORDINATE TRANSFORMATION $\ldots \ldots \ldots 5$

THE NODAL METHOD $\ldots \ldots \ldots \ldots \ldots \ldots \ldots \ldots \ldots \ldots \ldots \ldots \ldots \ldots$

RESULTS AND DISCUSSION $\ldots \ldots \ldots \ldots \ldots \ldots \ldots \ldots \ldots \ldots \ldots \ldots$

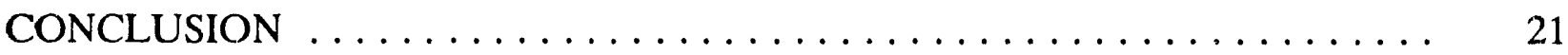

REFERENCES $\ldots \ldots \ldots \ldots \ldots \ldots \ldots \ldots \ldots \ldots \ldots \ldots \ldots \ldots \ldots \ldots \ldots \ldots \ldots \ldots, 22$

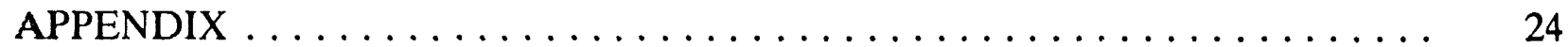

\section{DISCLAIMER}

This report was prepared as an account of work sponsored by an agency of the United States Government. Neither the United States Government nor any agency thereof, nor any of their employees, makes any warranty, express or implied, or assumes any legal liability or responsibility for the accuracy, completeness, or usefulness of any information, apparatus, product, or process disclosed, or represents that its use would not infringe privately owned rights. Reference herein to any specific commercial product, process, or service by trade name, trademark, manufacturer, or otherwise does not necessarily constitute or imply its endorsement, recommendation, or favoring by the United States Government or any agency thereof. The views and opinions of authors expressed herein do not necessarily state or reflect those of the United States Government or any agency thereof. 


\section{EXECUTIVE SUMMARY}

The work done under the Department of Energy Grant No. DE-FG0788ER12818, "Improved Deterministic Calculational Methods for Irregularly Shaped Shields" was completed on November 30, 1990. The research done under the grant included the development of the first deterministic neutron and radiation transport methods for irregularly shaped geometries. Three methods were developed by first introducing boundary-fitted-coordinate transformations for the transport equation, and then deriving compatible methods for the accurate and efficient numerical solution of the transformed transport equation. The first of these three was an $S_{N}$ method, developed principally as a reference against which to compare the other two methods, whose developments were the main objectives. The other two methods were a constant-constant nodal transport method and a linear-linear nodal transport method.

This final report summarizes the development, testing and evaluation of our boundary-fitted-coordinate $S_{N}$ method and of our boundary-fitted-coordinate noda] methods for neutron and radiation transport. The results obtained show that for given accuracy requirements the constant-constant nodal method is computationally the most efficient of the three numerical transport methods developed for irregularly shaped shields. More generally, they show that the use of boundary-fitted-coordinate transformations in conjunction with the compatible nodal transport methods we have developed comprises a practical approach to the deterministic calculation of neutron and radiation transport in irregular geometries. Thus these new nodal methods provide efficient techniques for practical deterministic calculations of particle transport in irregular geometries, as alternatives to costly Monte Carlo methods which traditionally have been required for such computations. 


\author{
IMPROVED DETERMINISTIC CALCULATIOIIAL METHODS FOR IRREGULARLY \\ SHAPED SHIELDS \\ Principal Investigator: \\ John J. Dorning \\ Whitney Stone Professor \\ Department of Iluclear Engineering and Engineering Physics
}

\title{
ABSTRACT
}

A new discrete nodal transport method has been developed for general twodimensional curvilinear geometry by using a boundary-fitted coordinate transformation from the general 'physical' coordinates to square 'computational' coordinates. The metrics which appear in the transformed transport equation are expanded using a simple polynomial function, and the angular divergence term is treated in the same way it is treated in $S_{N}$ methods for curved geometries. Because the metrics of the transformation depend upon the computational coordinates, the technical details of the formal development of the nodal method differ from those of ordinary nodal methods for rectangular geometry. However, the computational process in the transformed rectangular coordinate system is very simflar to that used in conventional discrete nodal transport methods. A discrete $S_{N}$ method also has been developed to solve the boundary-fitted coordinate transformed transport equation. Simple test problems for non-simple geometries were solved using the zeroth-order (constant-constant) nodal method, the first-order (1 inear-linear) nodal method, and the $S_{N}$ method for the same physical and computational grids. The results for the test problems studied showed that, for most performance criteria, the computational efficiency of the zeroth-order nodal method was the highest of the three methods. 


\section{IIIIROUUCIIOII}

Varlous nodal methods for solving the direction-discretized transport equation $(1-8)$, collectively referred to here as discrete nodal transport methods (DIIIH's), have been shown, since they first were developed in the late 70s, to be superfor in computational efficiency to the conventional discrete $S_{11}$ method. Although zeroth- and first-order multidimensional Uillil's have been developed and applied to fuel assembly, shielding, and well-logging problems, the ir development has been limited for the most part to Cartesian geometry $(1-5)$ with some extensions to cylindrical geometry(6-8), even though most practical problems arise in the contexts of far more complicated geometric configurations.

Recognizing the practical Importance of accommodating more complicated geometries, especially in realistic radiation shielding problems, nodal methods and $S_{f}$ methods have been developed to solve such problems in conjunction with boundary-fitted coordinate transformations. Thelr development, implementation, testing and initial evaluation is reported here. The coordinate transformations, from the general curvillinear geometry 'physical grid' to the square Cartesian geometry 'computational grid' - - or more precisely the inverse transformacions - are made via recently developed elliptic grid generation techniques (9-13). Such techniques have been used in conjunction with finitedifference solutions to the neutron diffusion equation(14); but liat application is similar to, and in many ways simpler than, the applications to fluid mechanics, where boundary-fitted coordinate transformations have been used extensively in conjunction with finite difference solutions to the transformed flulds equations. The situation here is more complicated for two important reasons. The first is because lie problems being solved are not simple diffusion or cont inuun flow problems; rather, they are radlation 
transport problems that require the inclusion of the direction vectors of the particles which adds significant complexities. The second is because, in the interest of computational efficiency, the transformed transport equations are being solved by nodal methods rather than only by a simple finite difierence or $S_{N}$ method (although an $S_{\mathcal{N}}$ method also is developed for comparison purposes); hence, the complications that arise from the space dependence of the transformation metrics must be addressed in the formulations of the nodal methods. The transformed transport equations are general curvilinear geometry transport equations in the transformed or computational spatial variables $(\xi, \eta)$. Hence, the spatial divergence terms include spatial differentiai operators with space-dependent coefficients (the metrics of the transformations), and there also are angular divergence terms in the transformed equations. Thus, there are two points which make the problem complicated in a general curved geometry. One is the metrics that exist in the spatial divergence term; the other is the angular divergence term. In the nodal methods, we expand the metrics using Legendre polynomials, and we treat the angular divergence term using the angular redistribution coefficients utilized in $S_{N}$ methods. In the $S_{N}$ method the metrics simply are treated as they are in $S_{N}$ methods for cylindrical and spherical geometrles. The transformed transport equation is solved on the computational grid using one or more of these three methods: the constant-constant $(C-C)$ nodal method (1.e., the discrete nodal method with constant node-surface and constant nodeIntertor spatial expansions of the discretized angular flux), the linearIinear $(L-L)$ nodal method, and the diamond-difference $S_{11}$ method.

After reviewing the orthogonal boundary-fitted coordinate transformation used in the next section, the development of the nodal methods is sunimarized in the third section. The results of the apulication of the three methods to two 
test problems are presented and discussed in the next-to-last section, and the conclusions are presented in the final section.

\section{THE BOUHDARY-FITTED COURUIHATE TRANSFORMATIOH}

Many numerical grid generation techiniques have been developed in recent years; however, because practical radiation transport problems usually involve bounded geometries, elliptic grid generation techniques seem particularly appropriate for them. The previously developed elliptic grid generation technique(12) that we have used begins from a set of two coupled elliptic partial differential equations for the computational coordinates $\xi$ and $\eta$ written in terms of the physical coordinates $x$ and $y$

$$
\xi_{\mathrm{xx}}+\xi_{\mathrm{yy}}=\mathrm{P}(\xi, \eta) ; \quad \eta_{\mathrm{xx}}+\eta_{\mathrm{yy}}=\eta(\xi, \eta)
$$

where the subscripts indicate differentiation with respect to these subscripts, and $P(\xi, \eta)$ and $Q(\xi, \eta)$ are control functions which can be specified to control the concentration of the grid in various physical regions. Since the computational grid $(\xi, \eta)$ actually is fixed first as a simple square grid and the structure of the physical grid $(x, y)$ actually is calculated, the dependent and independent variables in these equations must be interchanged so that $x(\xi, \eta)$ and $y(\xi, \eta)$ can be determined. The result is $(12)$

$$
\begin{aligned}
& a_{\xi \xi}-2 \beta x_{\xi \eta}+\gamma x_{\eta \eta}=-J^{2}\left(x_{\xi} \xi^{\prime}+x_{\eta} \eta^{\prime \prime}\right) \\
& a y_{\xi \xi}-2 \beta y_{\xi \eta}+\gamma y_{\eta \eta}=-J^{2}\left(y_{\xi} \xi^{\prime}+y_{\eta}^{\prime \prime}\right)
\end{aligned}
$$

where

$$
\begin{array}{ll}
a=\left(x_{\eta}\right)^{2}+\left(y_{\eta}\right)^{2}, & \beta=x_{\xi} x_{\eta}+y_{\xi} y_{\eta}, \\
\gamma=\left(x_{\xi}\right)^{2}+\left(y_{\xi}\right)^{2}, & J=x_{\xi} y_{\eta}-y_{\xi} x_{\eta} .
\end{array}
$$


This set of two coupled nonlinear elliptic partial differential equations is solved numerically for the boundary-fitted coordinates $x(\xi, \eta)$ and $y(\xi, \eta)$ in physical space for a specific set of control functions $P(x, y)$ and $Q(x, y)$ and specific boundary conditions that associate segments of the boundary of the physical domain with some or all the sides of the square computational domain. For certain choices of $P(x, y)$ and $Q(x, y)$, general elliptic grid generation transformations become orthogonal mappings. The use of such orthogonal mappings is convenient in radiation transport problems for two reasons. The first is that in this case, because certain rearranged combinations of the metrics which appear in the transformed transport equation depend upon only one of the transformed coordinates, it is possible to expand these quantities and develop various order self-consistent nodal methods. The second reason is that the most common transport boundary conditions such as specified incoming angular fluxes are most easily applied in the context of orthogonal transformations. Hence, we restrict the boundary-fitted coordinate transformations used here to the subclass that are orthogonal mappings(13). This is done by restricting the control functions to satisfy $P(x, y)=$ $(\alpha \gamma)^{-1 / 2}(\partial / \partial \xi)(f)$ and $Q(x, y)=(a \gamma)^{-1 / 2}(\partial / \partial \eta)(1 / f)$ where $f(x, y)$ is called the distortion function. Under these conditions, Eqs. (2) become

$$
\left(f x_{\xi}\right)_{\xi}+\left(\frac{1}{f} x_{\eta}\right)_{\eta}=0 ; \quad\left(f y_{\xi}\right)_{\xi}+\left(\frac{1}{T} y_{\eta}\right)_{\eta}=0 ; \quad f=(n / \gamma)^{1 / 2}
$$

Since an accurate representation of the boundary conditions usually is important in transport problems, retaining the flexibility of specifying the grid point distribution along the boundaries is desirable. Hence the "weak constraint" method(13) of solving Eqs. (4) was used. This method permits the complete specification of all the "boundary values" for $x(\xi, \eta)$ and $y(\xi, \eta)$ on 
the $(\xi, \eta)$ grid boundary. Then the distortion function $f(x, y)$ is determined along with a self-consistent solution to Eqs. (4). Thus, after complete specification of the boundary conditions, these equations were solved numerically via a finite difference method by iterating on the function $f(x, y)$ from an initial guess to determine the coordinate points $(x(\xi, \eta), y(\xi, \eta))$ in the physical plane which correspond to those in the computational plane $(\xi, \eta)$ at which the angular flux values, etc. are to be determined. Then the transport equation, transformed from physical spatial coordinates $(x, y)$ to computational spatial coordinates $(\xi, \eta)$, was solved $v$ ia the $C-C$ nodal method, the $L-L$ nodal method or the $S_{N}$ method, and the angular flux calculated for a point or region indexed by $\left(\xi_{j}, \eta_{j}\right)$ in the computational domain was plotted or tabulated (assigned) at the point or region corresponding to $\left(x\left(\xi_{j}, \eta_{j}\right)\right.$, $\left.y\left(\xi_{i}, \eta_{j}\right)\right)$ in the physical domain.

\section{THE HODAL METHOD}

The direction-discretized transport equation, for a one-group, twodimensional problem with two curvilinear coordinates, after transformation from the physical $(x, y)$ coordinates in a complicated physical domain to computational coordinates $(\xi, \eta)$ in a square domain, becomes

$$
\begin{aligned}
& \frac{1}{\left(x_{\xi}^{2}+y_{\xi}^{2}\right)^{1 / 2}\left(x_{\eta}^{2}+y_{\eta}^{2}\right)^{1 / 2}}\left[\mu \frac{\partial}{\partial \xi}\left(\left(x_{\eta}^{2}+y_{\eta}^{2}\right)^{1 / 2} N\right)+\nu_{\partial \eta}^{\partial}\left(\left(x_{\xi}^{2}+y_{\xi}^{2}\right)^{1 / 2} N\right)+\right.
\end{aligned}
$$

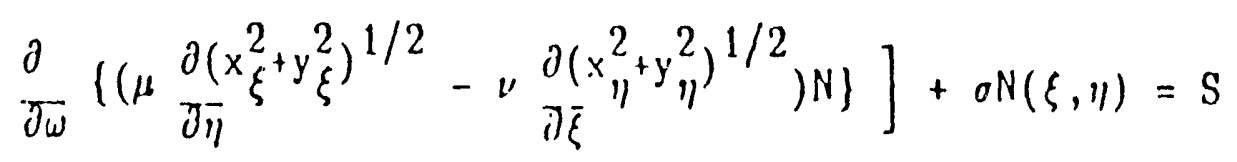

(Since $\eta$ is used traditionally both for a computational coordinate and a direction cosine, $\nu$ has been used for the latter here.) The detalled development of Eq. (5), the two-dimensional transport equation in general 
curvilinear coordinates, needed here to accommodate general boundary-fitted coordinate transformations is given in the appendix which very closely follows Ref. 15, adding to that work only its explicit completion for the general twodimensional case.

The development of the nodal methods begins by decomposing the square computational domain into computational volume elements. Equation (5) is then transformed from global computational coordinates $(\xi, \eta)$ to local coordinates $\left(\xi^{\prime}, \eta^{\prime}\right),-1 \leq \xi^{\prime}, \eta^{\prime} \leq+1$, using the simple transformations

$$
\xi=(\Delta \xi / 2) \xi^{\prime}+\xi_{I} ; \quad \eta=(\Delta \eta / 2) \eta^{\prime}+\eta_{J}
$$

where

$$
\begin{array}{ll}
\xi_{\mathrm{I}}=\left(\xi_{\mathrm{i}+1}+\xi_{\mathrm{i}}\right) / 2 ; & \Delta \xi=\xi_{\mathrm{i}+1}-\xi_{\mathrm{i}} \\
\eta_{\mathrm{J}}=\left(\eta_{\mathrm{j}+1}+\eta_{\mathrm{j}}\right) / 2 ; & \Delta \eta=\eta_{\mathrm{j}+1}-\eta_{\mathrm{j}}
\end{array}
$$

The transport equation in these local coordinates $\left(\xi^{\prime}, \eta^{\prime}\right)$ then is multiplied by $\Delta \xi \Delta \eta$ yielding

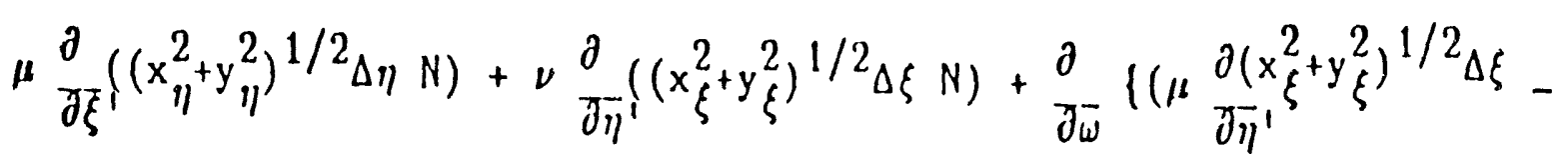

$$
\begin{aligned}
& \left.\left.\nu \frac{\partial\left(x_{\eta}^{2}+y_{\eta}^{2}\right)^{1 / 2} \Delta \eta \eta}{\partial \xi}\right) N\right\}+\frac{1}{2}(o N-S)\left(\left(x_{\xi}^{2}+y_{\xi}^{2}\right)\left(x_{\eta}^{2}+y_{\eta}^{2}\right)\right)^{1 / 2} \Delta \xi \Delta \eta=0
\end{aligned}
$$

where the denominator in Eq. (5) also has been cleared. Next, the direction vector $\Omega$ (or angle cosines $\mu$ and $\nu$ ) is discretized, and the angular divergence -- the third term in Eq. (8) -- is treated using the angular redistribution approach normally employed in the $S_{N 1}$ metliod $(16)$. The quantities $\left(x_{\eta}^{2}+y_{\eta}^{2}\right)^{1 / 2} \Delta \eta$ and $(x \xi+y \xi)^{1 / 2} \Delta \xi$.- incorrectly written in Ref. 17 in which $\Delta \eta$ and $\Delta \xi$ were inadvertently interchanged -. are expanded within each computational element 
using Legendre polynomials of the single variable $\xi^{\prime}$ or $\eta^{\prime}$ upon which each respectively tepends. These expansions are then truncated at first order, and with $a$ and $b$ defined as the zeroth and first coefficients in the Legendre expansion of the first of these quantities and $c$ and $d$ as the zeroth and first coefficients in the expansion of the second quantity, Eq. (8) becomes

$$
\begin{aligned}
& \mu_{\mathrm{m}, \ell} \ell_{\partial \xi}^{\partial}((a+b \xi) N)+\nu_{m, \ell} \frac{\partial}{\partial \eta}((c+d \eta) N)+\frac{\Delta \Lambda}{2 \eta} i\left(a_{m+1 / 2} N_{m+1 / 2}-a_{m-1 / 2} N_{m-1 / 2}\right) \\
& +{ }_{2 \pi}^{\Delta H} j\left(\beta_{\ell+1 / 2^{N} \ell+1 / 2}-\beta_{\ell-1 / 2^{N} \ell-1 / 2}\right)+\frac{1}{2}(\sigma N-S)(a+b \xi)(c+d \eta)=0
\end{aligned}
$$

where the primes $\left({ }^{\prime}\right)$ on $\xi^{\prime}$ and $\eta^{\prime}$ have been dropped so that $\xi$ and $\eta$ henceforth represent the local spatial computational coordinates, and the additional symbols introduced have the standard definitions used for them in the development of the $S_{N}$ method for curved geometries

$W=W_{m, l}$ : The area element on the unit directional sphere associated with direction vector $n_{\mathrm{m}, \ell}$.

$\alpha_{m}-1 / 2$ : The angular redistribution coefficient in the $i$-direction at the inner edge of the angular mesh denoted by $W_{m, \ell}$.

$\beta_{\ell}-1 / 2$ : The angular redistribution coefficient in the $j$-direction at the inner edge of the angular mesh denoted by $W_{m, \ell}$.

$\Delta A_{i}: \quad A_{i+1}-A_{i}$.

$A_{i}$ : The area element of the $i$ surface of a spatial computational element.

$\Delta B_{j}: \quad B_{j+1}-B_{j}$.

$B_{j}$ : The area element of the $j$ surface of a spatial computational element.

The zeroth-order $(C-C)$ and first-order $(L-L)$ nodal methods are developed directly from Eq. (9) by obtaining expressions for the zeroth and first 
transverse moments of the outgoing angular fluxes on the surfaces of the computational elements. This is done by first transverse integrating Eq. (9) in one direction (e.g., the $\eta$-direction) within a computational element to obtain a one-dimensional transport equation, formally solving the resulting $\xi$ dependent equation analytically, and taking the limit as $\xi \rightarrow+1$ to arrive at the following expression for the outgoing angular flux on the right boundary of the square element in the computational plane

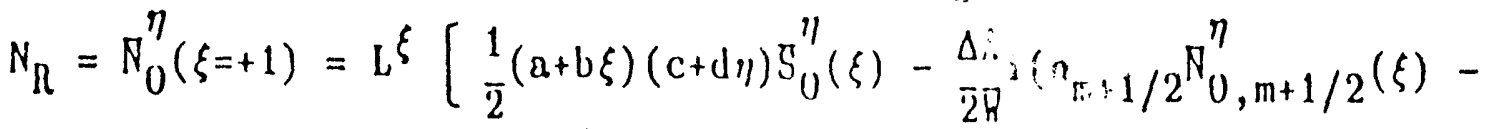

$$
\begin{aligned}
& \left.a_{m-1 / 2} \mathbb{R}_{0, m-1 / 2}^{\eta}(\xi)\right)-{ }_{2}{ }^{n} j\left(\beta_{\ell+1 / 2} N_{0, \ell+1 / 2}^{\eta}(\xi)-\beta_{\ell-1 / 2} \mathbb{R}_{0, \ell-1 / 2}^{\eta}(\xi)\right) \\
& \left.-\frac{\nu_{\mathrm{m}, \ell}}{2}[(\mathrm{c}+\mathrm{d} \eta) N(\xi, \eta)]_{\eta=-1}^{\eta=+1}-\frac{\sigma}{4} \mathrm{~d}(\mathrm{a}+\mathrm{b} \xi) N_{1}^{\eta}(\xi)\right]+L_{0}^{\xi} N_{0}^{\eta}(\xi=-1), \\
& \mu_{\mathrm{m}, \ell}, \nu_{\mathrm{m}, \ell}>0
\end{aligned}
$$

Here $2 \pi_{0}^{\eta}(\xi)$ is the zeroth spatial Legendre moment in the $\eta$ direction, within the computational element, of the flux in the direction $\left(\mu_{\mathrm{m}, \ell}, \nu_{\mathrm{m}, \ell}\right)$. The same quantity is indicated at the boundaries of $\left(\Delta \mu_{\mathrm{m}, l}, \Delta \nu_{\mathrm{m}, l}\right)$ by the additional subscripts $m \pm 1 / 2$ or $\ell \pm 1 / 2$. The integral operator $L \xi$ and the constant coefficient $L \xi$ in Eq. (10) are given by

$$
L^{\xi}=\int_{-1}^{+1} d \xi \frac{\exp \left\{-\int_{\xi}^{1}\left[\frac{\sigma c}{2 \mu}+\frac{b}{\left(a+\sqrt{5} \xi^{\top}\right)}\right] d \xi^{\prime}\right\}}{\mu(a+b \xi)} \cdot
$$

and

$$
L_{0}^{\xi}=\exp \left\{-\int_{-1}^{+1}\left[\frac{\sigma c}{2 \mu}+\frac{b}{(a+b \xi)}\right] d \xi\right\}
$$

To obtain the equation for the first spatial Legendre moment $\Pi_{1}^{\eta}(\xi)$ of the outgoing surface angular flux, the linear moment of Eq. (9) is taken in the $\pi$ 
direction, the one-dimensional equation thus generated is solved, and the result is evaluated at $\xi=+1$ yielding

$$
\begin{aligned}
& \theta_{R}=\pi_{1}^{\eta}(\xi=+1)=L^{\xi}\left[\frac{1}{2}(a+b \xi)(c+d \eta) S_{1}^{\eta}(\xi)-{ }_{i ̈ H} \Lambda_{i}\left(a_{m+1 / 2} N_{1, m+1 / 2}^{\eta}(\xi)-\right.\right. \\
& \left.a_{m-1 / 2} \mathbb{R}_{1, m-1 / 2}^{\eta}(\xi)\right)-\frac{\Delta B}{2 R} j\left(\beta_{\ell+1 / 2} \bar{N}_{1, \ell+1 / 2}^{\eta}(\xi)-\beta_{\ell-1 / 2} \mathbb{N}_{1, \ell-1 / 2}^{\eta}(\xi)\right) \\
& \left.-\nu_{\mathrm{m}, \ell}[\eta(\mathrm{c}+\mathrm{d} \eta) N(\xi, \eta)]_{\eta=-1}^{\eta=+1}+\nu_{\mathrm{m}, \ell}\left(2 \mathrm{c} \mathbb{N}_{0}^{\eta}(\xi)+\mathrm{dN} \mathbb{1}_{1}^{\eta}(\xi)\right)-\frac{\sigma}{2} \mathrm{~d}(\mathrm{a}+\mathrm{b} \xi) \mathbb{N}_{2}^{\eta}(\xi)\right] \\
& +L_{0}^{\xi} R_{1}^{\eta}(\xi=-1) \\
& \mu_{\mathrm{m}, \ell}, \nu_{\mathrm{m}, \ell}>0
\end{aligned}
$$

Here $\Pi_{2}^{\eta}(\xi)$ which arises from the combination of the first transverse Legendre moment with the linear term in the expansion of $(x \xi+y \xi)^{1 / 2} \Delta \xi$, is the simpie $\eta^{2}$ moment, not the second Legendre moment. These expresstons, Eqs. (10) and (12), are 1 formal analytical solutions for the zeroth and first $\eta$-moments of the outgoing angular fluxes at the right boundary of the computational element. Procedures similar to these, carried out on the $\xi$-integrated $\eta$-dependent equations, yield analogous expressions for $H_{T}=\Pi_{0}^{\xi}(\eta=+1)$ and $\theta_{T}=\Pi_{i}^{\xi}(\eta=+1)$. These are

$$
\begin{aligned}
& N_{T}=\mathbb{N}_{0}^{\xi}(\eta=+1)=L^{\eta}\left[\frac{1}{2}(a+b \xi)(c+d \eta) S_{0}^{\xi}(\eta)-\frac{\Delta \Lambda_{i}}{i} R\left(a_{m+1 / 2} \mathbb{R}_{0, m+1 / 2}^{\xi}(\eta)-\right.\right. \\
& \left.a_{m-1 / 2} \Gamma_{0, m-1 / 2}^{\xi}(\eta)\right)-\frac{\Delta B}{2} \nabla^{j}\left(\beta_{\ell+1 / 2} N_{0, \ell+1 / 2}^{\xi}(\eta)-\beta_{\ell-1 / 2} \nabla_{0, \ell-1 / 2}^{\xi}(\eta)\right) \\
& \left.-\frac{\mu_{\mathrm{m}, \ell}}{2}[(a+b \xi) N(\xi, \eta)]_{\xi=-1}^{\xi=+1}-\frac{\sigma}{\pi} b(c+d \eta) \Gamma_{1}^{\xi}(\eta)\right]+L_{0}^{\eta} \pi_{0}^{\xi}(\eta=-1), \\
& \mu_{\mathrm{m}, \ell}, \nu_{\mathrm{m}, \ell}>0
\end{aligned}
$$

and 


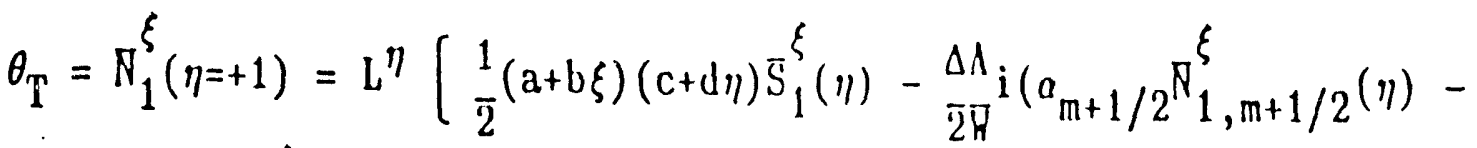

$$
\begin{aligned}
& \left.a_{\mathrm{m}-1 / 2} \mathbb{N}_{1, \mathrm{~m}-1 / 2}^{\xi}(\eta)\right)-{ }_{\overline{2} \boldsymbol{B}} \mathrm{B}\left(\beta_{\ell+1 / 2} \mathbb{N}_{1, \ell+1 / 2}^{\xi}(\eta)-\beta_{\ell-1 / 2} \mathbb{N}_{1, \ell-1 / 2}^{\xi}(\eta)\right) \\
& \left.-\mu_{\mathrm{m}, \ell}[\xi(\mathrm{a}+\mathrm{b} \xi) N(\xi, \eta)]_{\xi=-1}^{\xi=+1}+\mu_{\mathrm{m}, \ell}\left(2 \mathrm{a} \bar{N}_{0}^{\xi}(\eta)+\mathrm{b} \pi_{1}^{\xi}(\eta)\right)-\frac{\sigma}{\overline{2}} \mathrm{~b}(\mathrm{c}+\mathrm{d} \eta) \mathbb{N}_{2}^{\xi}(\eta)\right) \\
& +L_{0}^{\eta} \mathbb{N}_{1}^{\xi}(\eta=-1) \text {, } \\
& \mu_{\mathrm{m}, \ell}, \nu_{\mathrm{m}, \ell}>0
\end{aligned}
$$

where the integral operator $L^{\eta}$ and the constant $L B$ are given by

$$
\begin{aligned}
& L^{\eta}=\int_{-1}^{+1} d \eta \frac{\exp \left\{-\int_{\eta}^{1}\left[\frac{\sigma a}{2 \nu}+\frac{d}{\left(c+d \eta^{\prime}\right)}\right] d \eta^{\prime}\right\}}{\nu(c+d \eta)} . \\
& L_{0}^{\eta}=\exp \left\{-\int_{-1}^{+1}\left[\frac{\sigma a}{2 \nu}+\frac{d}{(c+d \eta)}\right] d \eta\right\}
\end{aligned}
$$

The integrals, that arise as a result of the two integral operators $L \xi$ and $L^{\eta}$ in these expressions, are evaluated by expanding the spatial variations of the node transverse-surface fluxes $N(\xi, \pm 1)$ and $N( \pm 1, \eta)$ and the node-interior flux in Legendre polynomials through first order in $\xi$ and $\eta$. For example,

$$
\begin{aligned}
& N(1, \eta)=N_{R}+(3 / 2) \theta_{R} \eta+\ldots \\
& N(\xi, 1)=N_{T}+(3 / 2) \theta_{T} \xi+\ldots
\end{aligned}
$$

and

$$
N(\xi, \eta)=(1 / 4) \overline{\bar{N}}_{00}+(3 / 4) \overline{\bar{N}}_{10} \xi+(3 / 4) \overline{\bar{N}}_{01} \eta+(9 / 4) \overline{\bar{N}}_{11} \xi \eta+\ldots
$$


or

$$
\begin{aligned}
& 2 N_{0}^{\eta}(\xi)=(1 / 2) \overline{\bar{N}}_{00}+(3 / 2) \overline{\bar{N}}_{10} \xi+\ldots \\
& R_{1}^{\eta}(\xi)=(1 / 2) \overline{\bar{N}}_{01}+(3 / 2) \overline{\bar{N}}_{11} \xi+\ldots
\end{aligned}
$$

and

$$
\Gamma_{2}^{\eta}(\xi)=(1 / 6) \overline{\bar{N}}_{00}+(1 / 2) \overline{\bar{N}}_{10} \xi+\ldots
$$

also is used. The double moments $\overline{\mathrm{n}}_{00}, \overline{\mathrm{n}}_{10}, \overline{\mathrm{n}}_{01}$ are obtained from the balance equation Eq. (9), by taking the corresponding moments. The double linear moment $\overline{\mathrm{F}}_{11}$ is neglected since it is second order.

The double zeroth moment equation is obtained by integrating Eq. (9) over the computational element in both the $\xi$ and $\eta$ directions which gives

$$
\begin{aligned}
& \mu\left\{(a+b) N_{R}-(a-b) N_{L}\right\}+\nu\left\{(c+d) N_{T}-(c-d) N_{B}\right)+\frac{\Delta \Lambda_{i}}{T}\left(a_{m+1 / 2} \overline{\bar{N}}_{00, m+1 / 2}-\right. \\
& \left.a_{m-1 / 2} \overline{\bar{N}}_{00, m-1 / 2}\right)+\frac{\Delta \mathrm{B}}{\mathrm{h}} \mathrm{j}\left(\beta_{\ell+1 / 2} \overline{\bar{N}}_{00, \ell+1 / 2}-\beta_{\ell-1 / 2} \overline{\bar{N}}_{00, \ell-1 / 2}\right)+ \\
& \sigma\left(a c \overline{\bar{N}}_{00}+b c \overline{\bar{N}}_{10}+a d \overline{\bar{N}}_{01}\right) / 4=\left(a c \overline{\bar{S}}_{00}+b c \overline{\bar{S}}_{10}+a d \overline{\bar{S}}_{01}\right) / 4
\end{aligned}
$$

The first $\xi$-moment zeroth $\eta$-moment equation is obtained by multiplying Eq. (9) by $\xi$ and again integrating over the computational element in both directions which yields.

$$
\begin{aligned}
& \mu\left\{2(a+b) N_{R}+2(a-b) N_{L}-a \bar{N}_{00}-b \overline{\bar{N}}_{10}\right\}+\nu\left\{(c+d) \theta_{T}-(c-d) \theta_{13}\right\} \\
& +\frac{\Delta \Lambda_{i}}{i}\left(\alpha_{m+1 / 2} \overline{\bar{N}}_{10, m+1 / 2}-a_{m-1 / 2} \overline{\bar{N}}_{10, m-1 / 2}\right)+\frac{\Delta l]}{2 R} j\left(\beta_{\ell+1 / 2} \overline{\bar{N}}_{10, \ell+1 / 2}\right. \\
& \left.-\beta_{\ell-1 / 2} \overline{\bar{N}}_{10, \ell-1 / 2}\right)-\sigma\left(b c \overline{\bar{N}}_{00}+3 a c \overline{\bar{N}}_{10}+2 b d \overline{\bar{N}}_{01}\right) / 6= \\
& \left(b c \bar{S}_{U v}+3 a c \overline{\bar{S}}_{10}+2 b d \bar{S}_{01}\right) / 6
\end{aligned}
$$


Finally, the zeroth $\xi$-moment first $\eta$-moment equation is obtained by multiplying Eq. (9) by $\eta$ and integrating over the element which gives

$$
\begin{aligned}
& \mu\left\{(\mathrm{a}+\mathrm{b}) \theta_{\mathrm{R}}-(\mathrm{a}-\mathrm{b}) \theta_{\mathrm{L}}\right\}+\nu\left\{2(\mathrm{c}+\mathrm{d}) \mathrm{N}_{\mathrm{I}}+2(\mathrm{c}-\mathrm{d}) \mathrm{N}_{\mathrm{B}}-\mathrm{c} \overline{\mathrm{N}}_{00}-\mathrm{dN_{01 }}\right\} \\
& +\frac{\Delta \Lambda_{i}}{2 R}\left(a_{m+1 / 2} \overline{\bar{N}}_{01, m+1 / 2}-a_{m-1 / 2} \overline{\bar{N}}_{01, m-1 / 2}\right)+\frac{\Delta l\}}{2 R} j\left(\beta_{\ell+1 / 2} \overline{\bar{N}}_{01, \ell+1 / 2}\right. \\
& \left.-\beta_{\ell-1 / 2} \overline{\bar{N}}_{01, \ell-1 / 2}\right)-\sigma\left(\operatorname{adN}_{00}+3 a c \bar{N}_{01}+2 \operatorname{bdv}_{10}\right) / 6= \\
& \left(\operatorname{adS} \bar{S}_{00}+3 a c \overline{\bar{S}}_{01}+2 b d \bar{S}_{10}\right) / 6
\end{aligned}
$$

These three balance equations are used with the surface flux moment equations, Eqs. (10), (12), (13) and (14), which together yield a closed system of equations.

In the C-C DNTM, we keep the constant terms in the surface and interfor flux expansions in Eq. (16). For the three unknowns $N_{R}, N_{T}$ and $\vec{H}_{00}$, we use respectively the expressions for the zeroth moments of the outgoing surface fluxes, Eqs. (10) and (13), and the double zeroth moment of the balance equation, Eq. (17). In the L-L DHIM, we keep the constant and linear terms in the surface and interior flux expansions in Eq. (16). For the seven unknowns $N_{R}, N_{T}, \theta_{R}, \theta_{T}, \bar{P}_{00}, \overline{\mathrm{H}}_{10}$ and $\overrightarrow{\mathrm{P}}_{01}$, we use the expressions for the zeroth and first moments of the outgoing surface fluxes, Eqs. (10), (13), (12) and (14), and the three double moment balance equations, Eqs. (17), (18) and (19). We employ the method used in TWOTRAN(16) to estimate the unknown interior flux moments at the outer edge of the angular mesh. That is, to initialize the angular sweep we set the interior moments of the flux at the first discretized angle equal to those at the inner edge of the first discretized angular mesh ce11. After that, the diamond difference approximation is used to estimate the values at the outer edge of an angular mesh cell in terms of the values at the 
center and inner edge of that cell.

The solution algorithm is similar to that used in Cartesian geometry nodal methods except that for certain physical geometries iterations must be performed because we must use initial guesses for the 'incoming' angular fluxes at an internal 'boundary', rather than known boundary values for true incoming angular fluxes at an exterior boundary.

The detalled development of the $S_{11}$ method for solving Eq. (5), the boundary-fitted coordinate transformed transport equation, will be omitted because $i t$ is analogous to that used for spherical geometry(16) in which there also are two curved coordinates.

\section{RESULTS AIID DISCUSSION}

In order to validate the boundary-fitted coordinate nodal methods, they were applied to special-case test problems in conventional right circular cylindrical geometry $(8)$ prior to the applications to problems in more complicated geometries reported here. Those studies(8) established that the metrics could be treated in the nodal methods as they are treated here and that the angular divergence term could be adequately approximated in the nodal methods via an angular redistribution term, as it is approximated in $S_{N}$ methods. They also demonstrated that the computational efficiency of the $C-C$ nodal method was greater than those of the $S_{f 1}$ method and the $L-L$ nodal method for cylindrical geometry, at least for the test problems studied. This is in contrast to conclusions made for an earlier cylindrical geometry $\mathrm{C}$ - $\mathrm{C}$ nodal method(6), in which the angular divergence term was treated via a transverse integration procedure analogously to the way the spatial derivatives are treated in nodal transport methods. There, the authors concluded that the $C-C$ nodal method was less efficlent than the $S_{11}$ method because the nodal-method- 
like treatment of the angular divergence ierm resulted in the need to solve nodal equations for two-dimensional cylindrical geometry that require computational effort which is comparable to that required for three-dimensional Cartesian geometry nodal equations $(6)$. Hence, the approach used here to treat the angular divergence term in the boundary-fitted coordinate transformed transport equation not only worked for the cylindrical geometry transport equation, but it also was more efficient than that clearly more elegant but less practical alternative.

The first problem solved that was not a 'conventional geometry' special case was one made up of two cylinders, the smaller one located eccentrically Inside the larger one. This problem involves two curvilinear coordinates and is meant to be an extreme idealization of the salient features of the problem of shielding an eccentrically located cylindrical shell radiation source. Although it is not cylindrically symetric, there is left-right symmetry; hence, the problem was solved using half the physical domain with a reflective boundary condition at the plane of symmetry. The boundary-fitted coordinate grid on the physical domain for an $80 \times 80$ 'radial'(I)-'angular' (J) grid is shown in Fig. 1 for the region exterior to the inner cylinder. The corresponding computational grid, from which it was generated, of course is a simple $80 \times 80$ square grid. None of the coarser boundary-fitted coordinate grids used in the studies reported here was generated directly; rather, they were derived from the $80 \times 80$ grid by successively combining two or four cells into one to obtain the next coarser grid. This was done by connecting the four resulting cell corner points on the new coarser grid by straight lines for grid illustration purposes, but by preserving cell volume and cell surface area for computational purposes. The one-energy-group problem was solved in the S-2 approximation with $\mu_{1}=-\mu_{2}=\nu_{1}=-\nu_{2}=-0.577350269$ and equal weights $w=\pi / 2$. The 
radii of the two cylinders were $14 \mathrm{~cm}$ and $4 \mathrm{~cm}$, and the center of the latter was $6 \mathrm{~cm}$ below the center of the former. The cross sections in the outer region were $\Sigma_{\mathrm{a}}=1.00 \mathrm{~cm}^{-1}$ and $\Sigma_{\mathrm{S}}=0.25 \mathrm{~cm}^{-1}$. The inner cylinder was comprised of a 'perfect absorber' (all particles that enter are absorbed). A unit (per cm) half-range isotropic (outward-directed) source was located on the circumference of the inner cylinder.

Some of the results for this test problem are summarized in Table 1 in which the computer leakages from the outer cylinder are compared. Since both Cartesian geometry and cylindrical geometry approximations to a problem like this have errors that are difficult to quantify, the reference solution for the leakage was obtained from an h-squared extrapolation of the $40 \times 40$ and $80 \times 80 S_{N}$ computed values. Although this might bias the comparison a little in favor of the $S_{\mathcal{N}}$ method, that bias is very small since the h-squared extrapolation of the $20 \times 20$ and $40 \times 40$ values computed by the $C-C$ nodal method was only about 0.002 (0.25 percent) lower. Notwithstanding some small error in the reference value, it is clear from Table 1 that the C-C DHIM satisfies a given error criterion on the leakage in less iterition time than either the $S_{N}$ method or the L-L DNIM. For example, the $20 \times 20 \mathrm{C}-\mathrm{C}$ nodal calculation $(2.45 \mathrm{sec})$ leads to an error (2.54 percent) between those that result in the $40 \times 40 \mathrm{~S}$ f calculation $(5.67 \mathrm{sec}$, -4.69 percent) and the $80 \times 80 S_{f f}$ calculation $(24.42 \mathrm{sec},-1.20$ percent); and the error in the $40 \times 40 \mathrm{C}-\mathrm{C}$ nodal calculation (10.16 sec, -0.65 percent) is less than that in the $80 \times 80 S_{N}$ calculation $(24.42 \mathrm{sec},-1.20$ percent). At the other extreme, for looser error criteria, the $5 \times 5$ C-C nodal calculation $10.16 \mathrm{sec}$, 15.38 percent) leads to a smaller error than the $20 \times 20 \mathrm{~S}$ N calculation (1.71 sec, -17.86 percent) in considerably less time. The leakage calculated using the $L-L$ nodal method on the $10 \times 10$ and $20 \times 20$ meshes for this problem were only slightly more accurate than those obtained using the $C$ - $C$ nodal method on the 
same meshes and the computation times were very dramatically larger. This is because it is necessary to invert a $7 \times 7$ matrix to calculate the outgoing angular fluxes from each node in the present $L-L$ nodal method formulation. Methods of circumventing this problem are being explored. However, if a significant improvement in the accuracy of the $L-L$ DNTM over that of the $C-C$ DNTM cannot be demonstrated for other test problems, there is little practical motivation to doing this. The error in the leakage in the $5 \times 5 \mathrm{~L}-\mathrm{L}$ DNTM calculation is larger than that in the $5 \times 5 \mathrm{C}-\mathrm{C}$ DNTM calculation; however, this may be due to the fact that on such a coarse mesh the L-L DIITH yields a negative flux in some nodes whereas the $C-C$ DNTM does not.

Additional results for this first test problem are shown in Figs. 2-5. Calculations were done for this problem using the $C-C$ nodal and $S_{\| f}$ methods on a sequence of grids in which the spatial 'angular' mesh (J) was fixed at 40 . This was done because, as one would expect, the spatial flux distributions are less sensitive to grid refinement in this direction than in the 'radial' direction. The 'radial' traverses, along the $j=39$ mesh boxes of a $\mathrm{J}=40$ mesh (1.e., a little to the right of vertically upward), of the angular fluxes at the outer surfaces of the computational elements in the direction to the left of the outward normal at the element surface, computed on three $S_{N}$ grids $(40 \times 40,80 \times 40,120 \times 40)$ and on three C-C nodal grids $(20 \times 40,40 \times 40,80 \times 40)$ are shown in Fig. 1. The $40 \times 40 S_{N}$ results osclllate falrly dramatically over the outer two-thirds of the radial mesh and the distribution near the surface, even if smoothed, would be in substantial error. As the 'radial' mesh is refined to 80 and 120, these $S_{\text {f }}$ angular fluxes become more accurate, but the oscillations, although less severe, are still significant. Conversely, the angular fluxes that result from the $40 \times 40$ and $80 \times 40$ C-C nodal calculations are smooth and appear to be quite accurate, agreeing reasonably well with the $120 \times 40 \mathrm{~S}_{N}$ 
results except, of course, that they do not exhibit the erroneous oscillations that plague the $S_{N}$ method on these 'insufficiently fine' $S_{N}$ meshes. Moreover, even the $20 \times 40$ C $-C$ nodal results appear to be quite accurate in addition to being smooth. Finally, the spatial distributions of the angular flux that result from the $\mathrm{C}-\mathrm{C}$ nodal calculations appear to be converging smoothly and quickly as a function of the 'radial' (I) mesh. Although those that result from the $S_{N}$ calculations also appear to be converging, due to the oscillations it is not clear how smooth or rapid this convergence is. The 'radial' transverses along the same mesh boxes of the total scalar fluxes resulting from the same six computations are shown in Fig. 3. They have similar characteristics to those of the angular fluxes that were just discussed.

Finally, the angular fluxes at the surface of the outer cylinder in the direction to the left of the outward normal, and the outgoing fluxes at this surface (which also are the total scalar fluxes there) that resulted from the three $S_{N}$ calculations and from the three $C-C$ nodal calculations are shown in Figs. 4 and 5. The fluxes are plotted in both figures as functions of position along the circumference of the outer cylinder starting from the bottom (i.e., as functions of the 'angular' (j) mesh). Naturally, the fluxes decrease as the top of the cylinder is approached since the optical distance from the source is maximum there. Just as in the two 'radial' (I nesh) traverses shown in Figs. 2 and 3 , both the angular and scalar fluxes that resulted from the $S_{N}$ calculations oscillate, here only in about the upper half of the 'angular' mesh, and those from the $C-C$ nodal method are smooth and appear to be converging smoothly and rapidly.

The second test problem studied was composed of the same eccentric twodimensional cylindrical geometry. In this case, however, the inner cylinder (region 1) was not a 'perfect' absorber; rather, it had total cross section $\Sigma$ ! 
- $2.40 \mathrm{~cm}^{-1}$ and an absorption cross section $\Sigma_{a}^{1}=2.0 \mathrm{~cm}^{-1}$. The cross sections in the outer region (region 2) were the same as those in the first test problem, as were all the dimensions. In this second problem, the source also was different. It was a unit (per $\mathrm{cm}$ ) half-range isotropic (inward-directed) source located on the circumference of the outer cylinder. As in the first test problem non-reentrant vacuum boundary conditions were used on the outer surface of the outer cylinder. This geometry and source arrangement is of interest as a crude representation of an eccentrically shielded detector or some inner chamber of a large facility. It also is relevant to the detection of neutrons and gamma rays returning to bore holes from underground formations in nuclear oil-well logging and uranium exploration. This one-energy-group problem was solved in the S-2 approximation using the same quadratures and weights used for the first test problem. The same grids also were used in region 2, and the grids used in region 1 were special cases of boundary-fitted coordinate grids -. uniform standard cylindrical $r$ - $\theta$ grids.

A brief summary of the results for this second test problem is presented in Table 2. Computed values for the total absorption in reglon 1, the quantity of principal interest, are tabulated for solutions obtained using the C-C DNTH and the $S_{N}$ method, each on five meshes. In this sequence of grids, the 'angular' (J) mesh was kept constant at twenty, a value that was determined to be adequate for these studies, and the inner 'radial' $\left(l_{1}\right)$ mesh was kept constant at forty, which also was determined to be adequate for the correct computation of the absorption in region 1. Because three mesh numbers were involved in this sequence of grids, and only one was varied, extrapolation to a reliable reference solution was not possible. Hence, a fine mesh $S_{N}$ result was used as the reference value which, from the systematic convergence of the errors for both the C-C DNIH and the $S_{1 /}$ method, appears to be a fairly good 
value. A quick glance at the errors in Table 2 shows that the $C-C$ nodal method yields extremely accurate values for the absorption in region 1 in this problem even with only 5 mesh points in the 'radial' direction in region 2 ; in fact, it ylelds a smaller error than the $S_{\text {N }}$ method does with forty mesi points in that direction in that region. The ratios of the computing times, $S_{N}$ to $C-C$ nodal, for comparable error criteria are not as impressive, however. This almost certainly is because refining the 'radial' mesh in the outer region (increasing $\left.I_{2}\right)$ does not significantly increase the total number of unknowns or the computation time, when $l_{1}$ is so large, until $I_{2}$ becomes falrly large. When the $S_{N}$ grid is sufficiently fine, e.g., $(40+40) \times 20$, the error is fairly small and these results also seem satisfactory. However, when the grid is coarse the $S_{N}$ error becomes quite large, with the exception of the $(40+10) \times 20$ case which appears to be fortuitous with a relatively small negative error that is quite out of pattern with the other $S_{N}$ results. On the other hand, one might argue that the very large errors that result from the $(40+5) \times 20$ and $(40+20) \times 20 S_{N}$ calculations are the unusual ones because of the negative fluxes that are obtained in those computations. In fact, negatfve fluxes plagued many of the boundary-fitted coordinate $S_{N}$ calculations summarized here with negative flux fix-ups on the angular fluxes being required even at the end of the final iteration. Conversely, the appearance of negative fluxes in the nodal methods was extremely rare, especially in the C-C DHIM.

\section{COHCLUSION}

In an effort to make possible improved deterministic transport computations for irregularly shaped radiation shields, reactor fuel pin cells and fuel bundles, and other applications such as nuclear well-logging, etc., we have begun the development of boundary-fitted coordinate transport methods. We have developed, encoded and tested constant-constant and linear-linear discrete 
nodal transport methods and an $S_{N}$ transport method, all three for boundaryfitted coordinate geometries. The results for the test problems studied show that these methods are accurate and have good computational efficiency. However, the numerical examples must be taken as preliminary, since a broad spectrum of test problems has not been studied using extensive sets of practical performance criteria. Nevertileless, the present results do establish the feasibility and value of combining discrete nodal transport methods and $S_{N}$ methods, developed here for general curvilinear coordinates, with boundaryfitted coordinate transformations using elliptic grid generation techniques for irregular geometries which so frequently are of practical interest. Both the boundary-fitted coordinate $S_{N}$ method and the boundary-fitted coordinate DNTM eventually should have their places in deterministic transport computations for irregular geometries.

\section{REFERENCES}

1. M. R. Wagner, "A Nodal Discrete-Ordinates Method for the Numerical Solution of the Multidimensional Transport Equation," Proc. ANS Top. Mtg. Comp. Methods in Nucl. Engr. 2, p. 4-117, Willilamsburg, VA (1979).

2. R. D. Lawrence and J. J. Dorning, "A Nodal Integral Transport Theory Method for Multidimensional Reactor Physics and Shielding Calculations," Proc. ANS Top. Mtg. 1980 Advances in Reactor Physics and Shielding, p. 240, Sun Valley, 10 (1980).

3. W. F. Walters and R. D. O'Dell, "Hodal Methods for Discrete-Ordinates Transport Problems in $(x, y)$-Geometry," Proc. ANS Top. Mtg. Advances Math. Methods for Solution of Nucl. Engr. Problems, p. 115, Munich, FRG (1981).

4. Y. Y. Azmy, Trans. Am. Hucl. Soc., 54, 350 (1987).

5. R. L. Childs and W. A. Rhoades, Trans, Am. Hucl. Soc., 50, 406 (1985).

6. T. Mishimura, K. Tada and H. Yokobori, J. Hucl. Scl. Tech., 19, No. 1, 80 (1982).

7. A. Badruzzaman, Irans. Am. Hucl. Snc., 56, 307 (1988).

8. S. T. Kim and J. J. Dorning, "A Discrete llodal Transport Method for Cylindrical Geometry," Irans. Am. Hucl. Soc., 57, 233 (1988). 
9. J. F. Thompson, and 2. U. Harsi, "Boundary-Fitted Coordinate System for Numerical Solution of Partial Differential Equations - A Review, " J. Comp. phys., 47 (1982).

10. C. W. Mastin and J. Thompson, "Transformation of Three-Dimensional Regions onto Rectangular Reglons by Elliptic Systems," Num. Mat., 29, 397-407 (1978).

11. J. L. Steger, "On Application of Body-Conforming Curvilinear Grids for Finite-Difference Solution of External Flow," in Numerical Grid Generation [Joe Thompson, ed.], Elsevier Science Publishing Co., New York, 1982.

12. J. F. Thompson, "General Curvilinear Coordinate Systems," in llumerical Grid Generation [Joe Thompson, ed.], Elsevier Science Publishing Co., New York, 1982 .

13. G. Ryskin and L. G. Leal, "Orthogonal Mapping," J. Comp. Phys., 50, 71-100 (1983).

14. S. Uchikawa, "Use of Boundary-Fitted Coordinate Transformation in Heutron Diffusion Calc:llations for Arbitrary Two-Dimensional Geometries," J. Nucl. Sci. Eng., 85, 36-44 (1983).

15. D. W. Drawbaugh, "The Tensor Form of the Neutron-Transport Equation with Application to Finite Differencing," Hucl. Sci. Eng., 44, 58-65, (1971).

16. K. D. Lathrop and F. W. Brinkley, "TWOTRAl-1I: An Interfaced, Exportable Version of the TWOTRAll Code for Two-Dimensional Transport," LA-4848-MS, Los Alamos National Laboratory, Los Alamos, NH, July 1973.

17. This report is an expanded version of the paper: S. T. Kim and J. J. Dorning, "Discrete llodal and $S_{\text {ff }}$ Transport Hethods for Boundary-Fitted Coordinate Geometries," in Advances in Nuclear Engineering Computation and Radiation Shielding, Vol. 1, pp. 2:1-2:15, American Nuclear Society, La Grange Park, IL (1989). 
APPENDIX: The Explicit Form of the Two-Dimensional Transport Equation in General Curvilinear Coordinates

A general development of the three-dimensional transport equation in general curvilinear coordinates already has been given(15). The final result obtained there for the transport equation in rectangular coordinates was

$$
\operatorname{div}(M N)+\text { oN }=S
$$

where $M$ is a contravariant vector in a five-dimensional space with a Riemannian metric. Since this equation is a five-dimensional tensor equation, it follows from the principle of convariance that it holds in any five-dimensional coordinate system.

The explicit forms of the transport equation in cylindrical coordinates and in spherical coordinates were derived from Eq. (A-1) in Ref. 15, but the analogous explicit form for general curvilinear coordinates was not. The general development there(15) ended with Eq. (A-1). Since the explicit form of the transport equation for the general two-dimensional curvilinear geometry case is needed here to accommodate the general two-dimensional boundary-fitted coordinate transformations, we continue from Eq. $(A-1)$ above, as given in Ref. 15, to develop this explicit form via steps completely analogous to those used to develop the explicit form for cylindrical geometry(15). Recalling the transport equation in rectangular coordinates, with $n_{x}-n_{0} l=$ $\left(1-5^{2}\right)^{1 / 2} \cos \left(\omega^{\prime}\right), n_{y}-n \cdot l=\left(1-5^{2}\right)^{1 / 2} \sin \left(\omega^{\circ}\right), n_{z}=n \cdot 2=5$, and $n_{x}^{2}+n_{y}^{2}+n_{z}^{2}=$ 1 ,

$$
n_{x} \frac{\partial N}{\partial x}+n_{y} \frac{\partial H}{\partial y}+n_{z} \frac{\partial N}{\partial z}+o H=S
$$

and the corresponding contravariant vector

$$
M_{\text {rect }}=\left(\Omega_{x}, \Omega_{y}, n_{z}, 0,0\right)
$$


we introduce the general curvilinear coordinates $(\xi, \eta)$ in the $(x, y)$ plane perpendicular to the straight $z$ coordinate to form the general coordinates $u^{1}=\xi, u^{2}=\eta, u^{3}=z, u^{4}=w$, and $u^{5}=5$. The resulting transformation equations from the curvilinear coordinates to the rectangular coordinates are

$$
\begin{aligned}
& x_{1}=x=x(\xi, \eta) \\
& x_{2}=y=y(\xi, \eta) \\
& x_{3}=z=z \\
& x_{4}=\omega^{\prime}=\omega+\theta \\
& x_{5}=5=5
\end{aligned}
$$

where $\omega$ is the angle formed by the projection of $\Omega$ onto the $(\xi, \eta)$ plane and the $\xi$-axis, and $\theta$ is the losal rotation angle of the orthogonal curvilinear coordinates $(\xi, \eta)$ with respect to tie fixed rectangular coordinates $(x, y)$.

Figure $f-1$ shows the angles $\omega, \omega^{\prime}$ and $\theta$ and the relationship between the curvilinear coordinates and the rectangular coordinates in the $(x, y)$ or $(\xi, \eta)$ plane.

The resulting Jacobian of the transformation from the curvilinear coordinates to the rectangular coordinates is

$$
\left|\frac{\partial x^{i}}{\partial u^{j}}\right|=\left|\begin{array}{ccccc}
x_{\xi} & y_{\xi} & 0 & A & 0 \\
x_{\eta} & y_{\eta} & 0 & B & 0 \\
0 & 0 & 1 & 0 & 0 \\
0 & 0 & 0 & 1 & 0 \\
0 & 0 & 0 & 0 & 1
\end{array}\right|
$$

and the Jacobian for the inverse transformation is 


$$
\left|\frac{\partial u^{i}}{\partial x^{j}}\right|=\left|\begin{array}{ccccc}
\xi_{x} & \eta_{x} & 0 & c & 0 \\
\xi_{y} & \eta_{y} & 0 & \nu & 0 \\
0 & 0 & 1 & 0 & 0 \\
0 & 0 & 0 & 1 & 0 \\
0 & 0 & 0 & 0 & 1
\end{array}\right|
$$

where

$$
\begin{array}{ll}
A=\frac{\partial}{\partial \xi}\left\{-\tan ^{-1}\left(-\frac{x_{\eta}}{y_{\eta}}\right)\right\} ; & B=\frac{\partial}{\partial \eta}\left\{-\tan ^{-1}\left(-\frac{x_{\eta}}{y_{\eta}}\right)\right\} \\
C=-\frac{\partial}{\partial x}\left\{\tan ^{-1}\left(\frac{\xi_{y}}{\xi_{x}}\right)\right\} ; & D=-\frac{\partial}{\partial y}\left\{\tan ^{-1}\left(\frac{\xi_{y}}{\xi_{x}}\right)\right\}
\end{array}
$$

The metric tensor $a_{i j}$ in the curvilinear system follows from

$$
a_{i j}=g_{k \ell} \frac{\partial x^{k}}{\partial u^{1}} \frac{\partial x^{\ell}}{\partial u^{j}}
$$

which yields

$$
\mathrm{J}^{2}=\left|a_{i j}\right|=\left(x_{\xi}^{2}+y_{\xi}^{2}\right)\left(x_{\eta}^{2}+y_{\eta}^{2}\right)-\left(x_{\xi} x_{\eta}+y_{\xi} y_{\eta}\right) ?
$$

From the contravariant vector in the rectangular system, we have

$$
H_{c u r v}^{i}=M_{r e c t}^{j} \frac{\partial u^{i}}{\partial x^{j}}=\left(\Omega_{x}, \Omega_{y}, \zeta, 0,0\right) \frac{\partial u^{i}}{\partial x^{j}}
$$

More explicitly, the components are 


$$
\begin{aligned}
& M_{\text {curv }}^{1}=\Omega_{x} \xi_{x}+\Omega_{y} \xi_{y} \\
& M_{\text {curv }}^{2}=\Omega_{x} \eta_{x}+\Omega_{y} \eta_{y} \\
& M_{\text {curv }}^{3}=\zeta \\
& M_{\text {curv }}^{4}=\Omega_{x} C+\Omega_{y} D \\
& M_{\text {curv }}^{5}=0 .
\end{aligned}
$$

In the curvilinear system, the divergence becomes

$$
\operatorname{liv}=\frac{1}{J} \frac{\partial}{\partial u^{1}}\left(\mathrm{JH}^{\mathrm{i}}\right)
$$

and the divergence form of the transport equation, Eq. $(A-1)$, in the curvilinear coordinates is

$$
\begin{aligned}
\frac{1}{J}[ & \frac{\partial}{\partial \xi}\left\{\mathrm{J}\left(\Omega_{x} \xi_{x}+\Omega_{y} \xi_{y}\right) N\right\}+\frac{\partial}{\partial \eta}\left\{\mathrm{J}\left(\Omega_{x} \eta_{x}+\Omega_{y} \eta_{y}\right) N\right\}+\frac{\partial}{\partial z}(J \zeta N) \\
& +\frac{\partial}{\partial \omega}\left\{J\left(\Omega_{x}\left(+\Omega_{y} D\right) N\right\}\right]+\sigma N=S .
\end{aligned}
$$

Now using the conditions for the orthogonal coordinate transformation

$$
\begin{aligned}
& \sin \theta=\frac{-y_{\xi}}{\left(x_{\xi}^{2}+y_{\xi}^{2}\right)^{1 / 2}}=\frac{x_{\eta}}{\left(x_{\eta}^{2}+y_{\eta}^{2}\right)^{1 / 2}} \\
& \cos \theta=\frac{x_{\xi}}{\left(x_{\xi}^{2}+y_{\xi}^{2}\right)^{1 / 2}}=\frac{y_{\eta}}{\left(x_{\eta}^{2}+y_{\eta}^{2}\right)^{1 / 2}} \\
& x_{\xi} x_{\eta}+y_{\xi} y_{\eta}=0
\end{aligned}
$$

and the general relationships between the coordinates

$$
\xi_{x}=y_{\eta} / J, \quad \xi_{y}=-x_{\eta} / J, \quad \eta_{x}=-y_{\xi} / J, \quad \eta_{y}=x_{\xi} / J .
$$


the contravariant terms multiplied by $\mathrm{J}$, written in Eq. (A-14) in terms of the derivatives of the curvilinear coordinates with respect to the rectangular coordinates, are rewritten in terms of the derivatives of the rectangular coordinates with respect to the curvilinear coordinates

$$
\begin{aligned}
& \mathrm{J}\left(\Omega_{\mathrm{x}} \xi_{\mathrm{x}}+\Omega_{\mathrm{y}} \xi_{\mathrm{y}}\right)=\mu\left(\mathrm{x}_{\eta}^{2}+\mathrm{y}_{\eta}^{2}\right)^{1 / 2} \\
& \mathrm{~J}\left(\Omega_{\mathrm{x}} \eta_{\mathrm{x}}+\Omega_{\mathrm{y}} \eta_{\mathrm{y}}\right)=\nu\left(\mathrm{x}_{\xi}^{2}+\mathrm{y}_{\xi}^{2}\right)^{1 / 2} \\
& \mathrm{~J} \zeta=\zeta\left(\mathrm{x}_{\eta}^{2}+\mathrm{y}_{\eta}^{2}\right)^{1 / 2}\left(\mathrm{x}_{\xi}^{2}+\mathrm{y}_{\xi}^{2}\right)^{1 / 2} \\
& \mathrm{~J}\left(\Omega_{\mathrm{x}} \mathrm{C}+\Omega_{\mathrm{y}} \mathrm{D}\right)=\mu \frac{\partial}{\partial \eta}\left(\mathrm{x}_{\xi}^{2}+\mathrm{y}_{\xi}^{2}\right)^{1 / 2}-\nu \frac{\partial}{\partial \xi}\left(\mathrm{x}_{\eta}^{2}+\mathrm{y}_{\eta}^{2}\right)^{1 / 2}
\end{aligned}
$$

where

$$
\mu \equiv\left(1-\zeta^{2}\right)^{1 / 2} \cos (\omega) ; \quad \nu \equiv\left(1-\zeta^{2}\right)^{1 / 2} \sin (\omega)
$$

Thus, finally the transformed transport equation in the two curvilinear coordinates $(\xi, \eta)$ and one straight coordinate $(z)$ becomes, in conservative form,

$$
\begin{aligned}
& \frac{1}{\left(x_{\xi}^{2}+y_{\xi}^{2}\right)^{1 / 2}\left(x_{\eta}^{2}+y_{\eta}^{2}\right) 1 / 2}\left[\mu \frac{\partial}{\partial \xi}\left(\left(x_{\eta}^{2}+y_{\eta}^{2}\right)^{1 / 2} N\right)+\nu \frac{\partial}{\partial_{\eta}}\left(\left(x_{\xi}^{2}+y_{\xi}^{2}\right)^{1 / 2} N\right)+\right.
\end{aligned}
$$

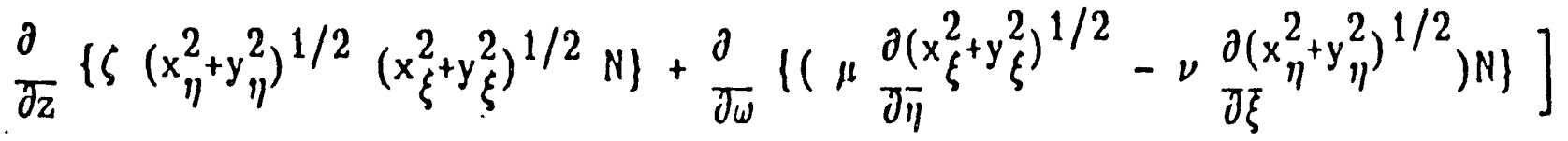

$$
\begin{aligned}
& +o N=S \text {, }
\end{aligned}
$$

For a system with geometry, sources, and boundary conditions uniform in the zdirection the derivative with respect to $z$ vanishes, and this reduces the transport equation for two-dimensional general curvilinear coordinates, Eq. in the main text, which subsequently is used there to accommodate the general boundary-fitted computational coordinates. 
In non-conservative form the transformed transport equation, Eq. (A-18), becomes

$$
\begin{aligned}
& \frac{\mu}{\left(x_{\xi}^{2}+y_{\xi}^{2}\right) 1 / 2} \frac{\partial N}{\partial \xi}+\frac{\nu}{\left(x_{\eta}^{2}+y_{\eta}^{2}\right)^{1 / 2}} \frac{\partial N}{\partial \eta}+\zeta \frac{\partial N}{\partial z}+ \\
& \frac{1}{\left(x_{\xi}^{2}+y_{\xi}^{2}\right) 1 / 2\left(x_{\eta}^{2}+y_{\eta}^{2}\right)} 1 / 2 \frac{\partial}{\partial \omega}\left\{\left(\mu \frac{\partial\left(x_{\xi}^{2}+y_{\xi}^{2}\right)^{1 / 2}-\nu}{\partial \eta} \frac{\left.\left.\partial\left(x_{\eta}^{2}+y_{\eta}^{2}\right)^{1 / 2}\right) N\right\}+o N=S .}{\partial \xi}\right.\right.
\end{aligned}
$$




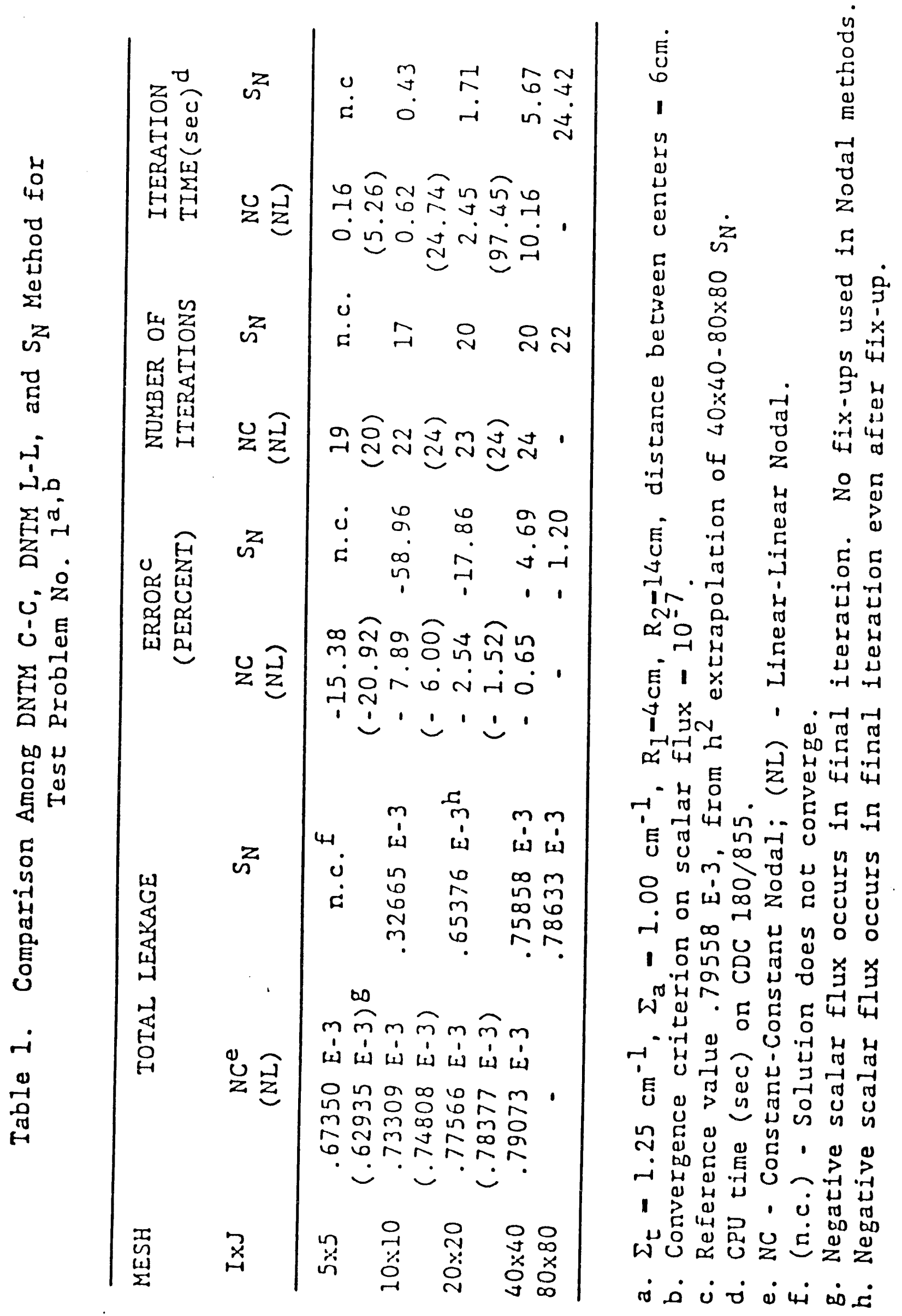




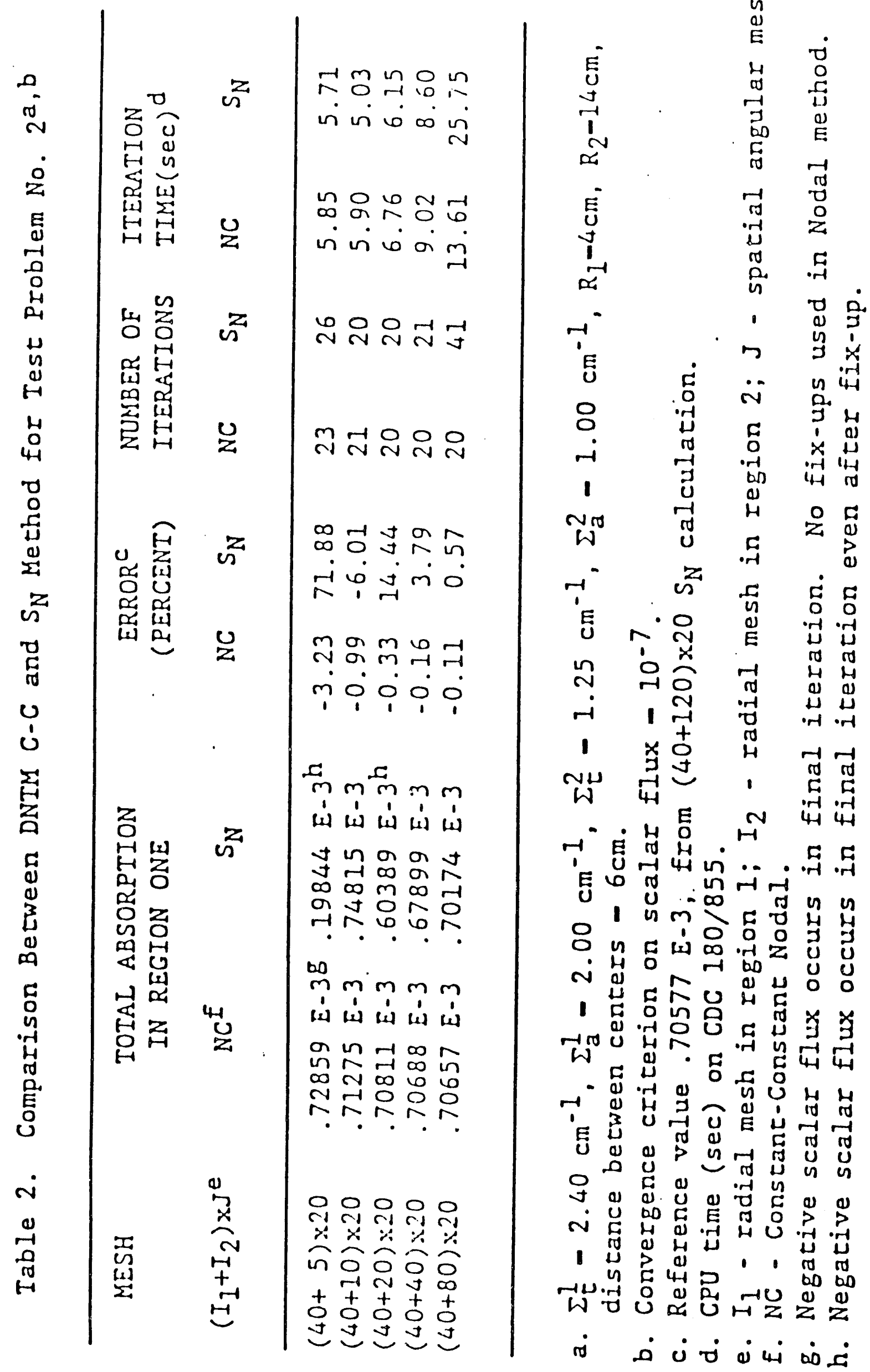




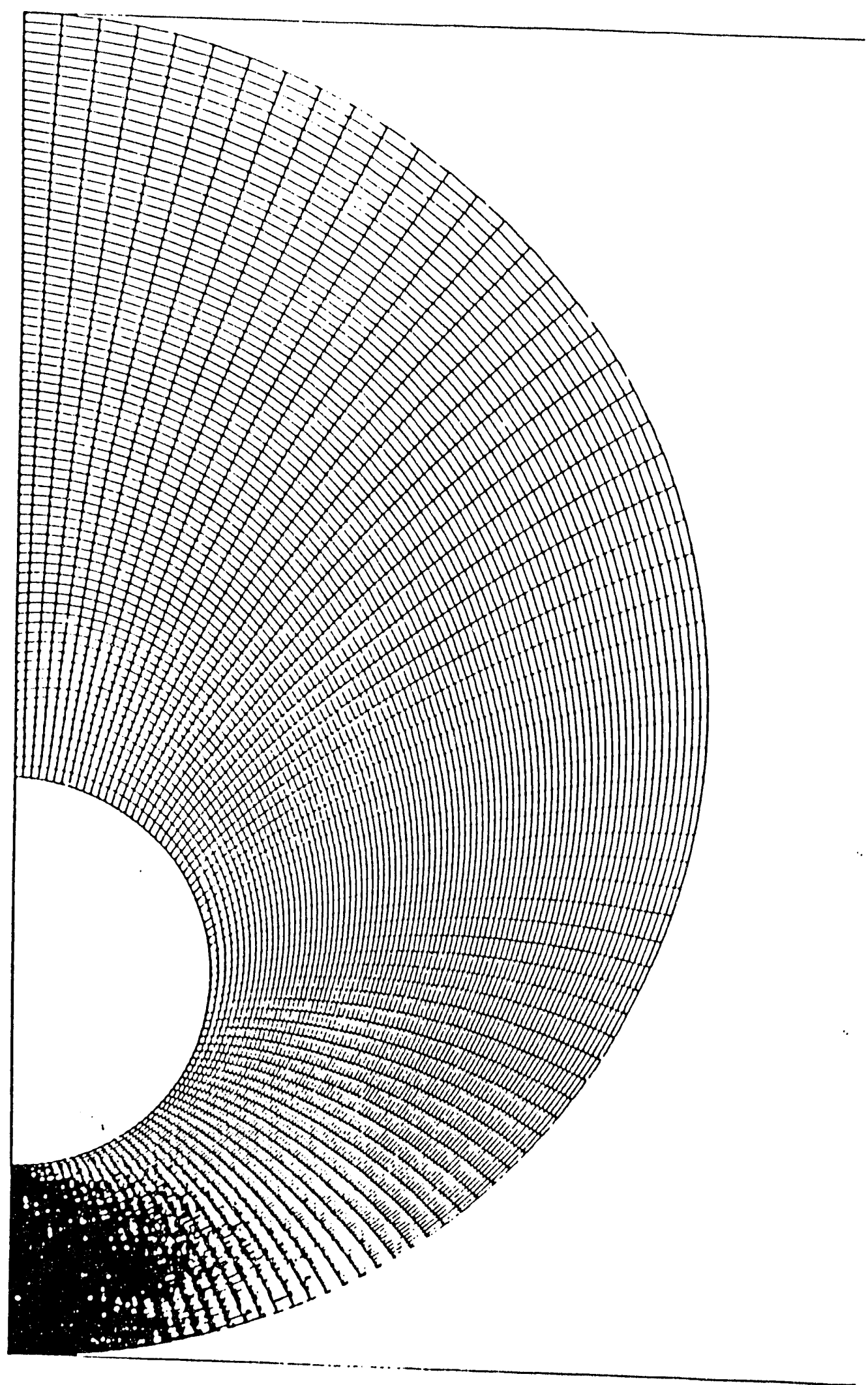

Figure 1. The $80 \times 80$ bommdary-111tnd courdluate grld lisnd In lest Problem llumber 1, and In Reglon 2 of lest Problein llumber 2 . 


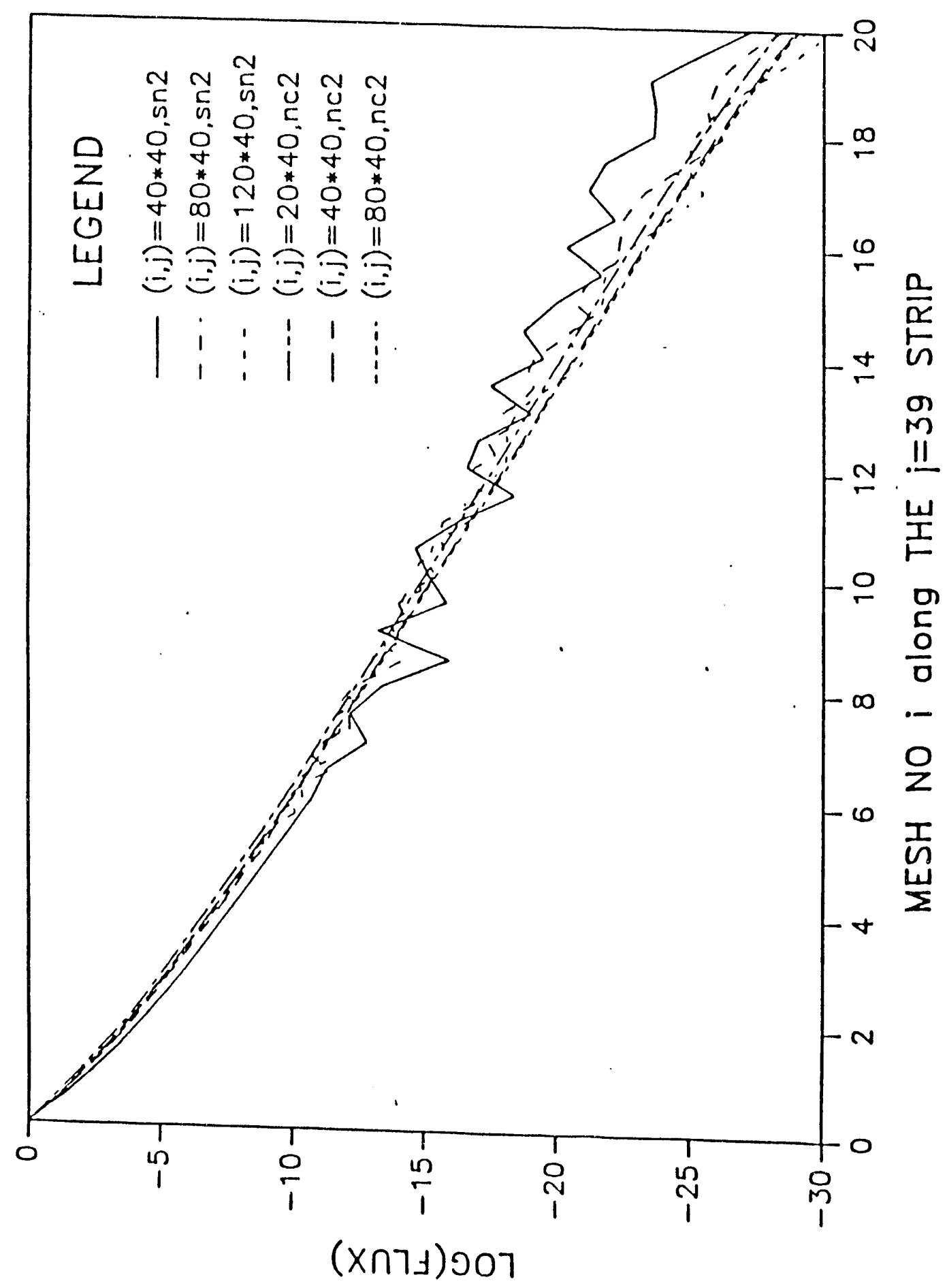

40

茛㟧

完造 的 a

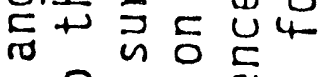

웅 음수

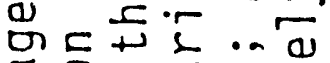

0 000

$1 \cdot r 4$ un $x$

a) 0 2क

$>$ on on

L 1 임

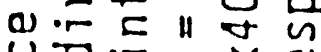

u. 0.0

m 4 \&

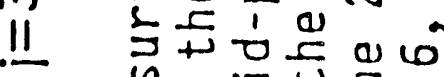

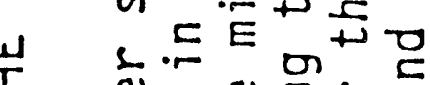

a

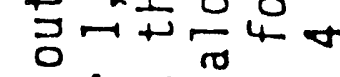

क 100

d क व

응ㅁำ

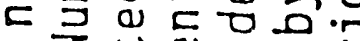

z

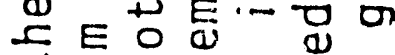

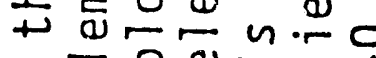

$4500 \frac{1}{2}$

0 \& n

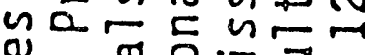

a 00

计E

๘

$>10$ 元

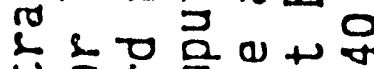

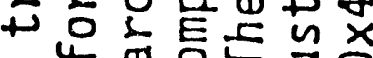

- 언르요

-

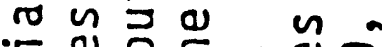

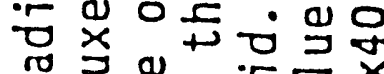

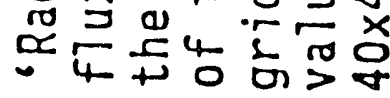

Ni

它 


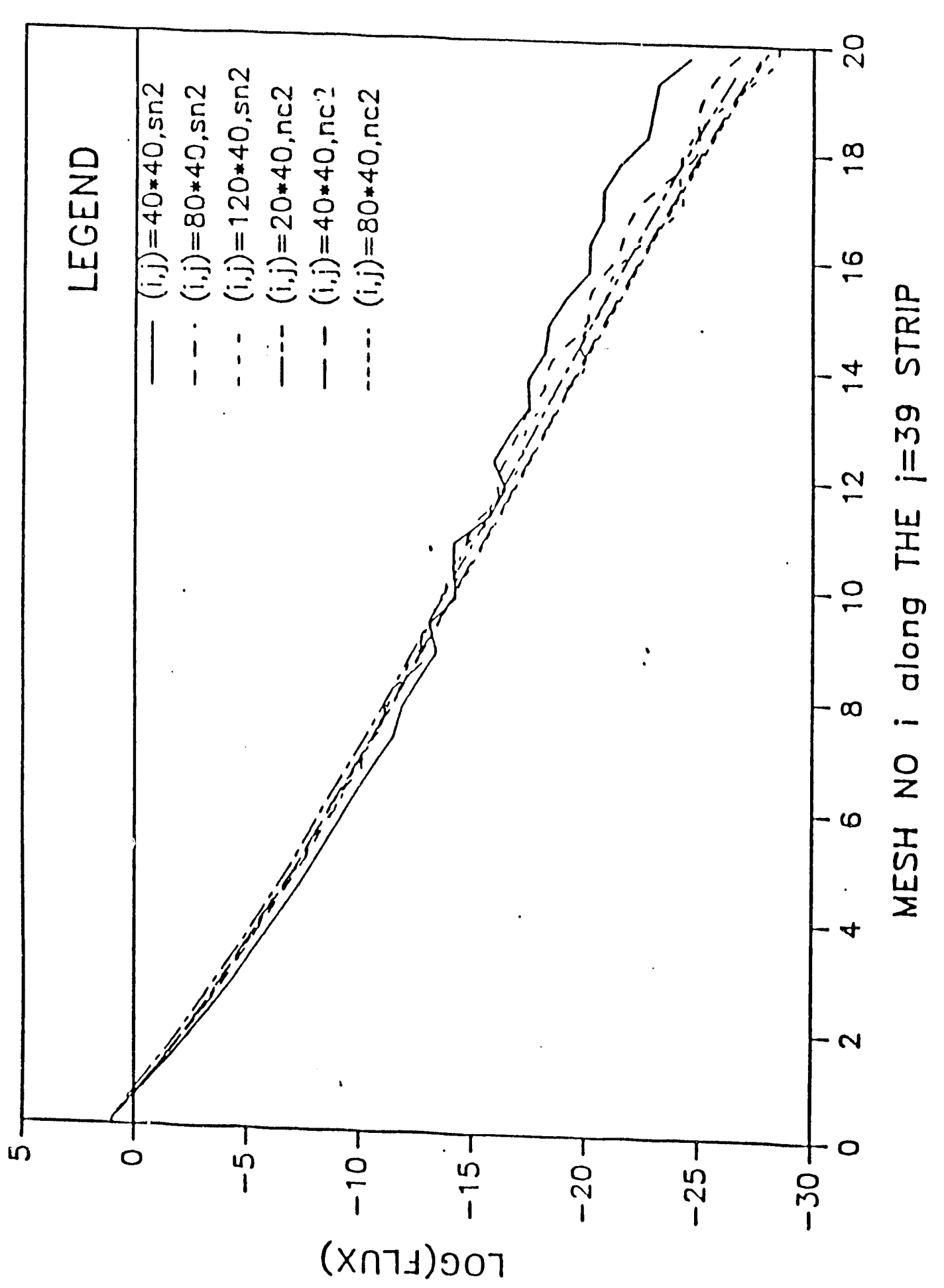

w in d i

a $n \cdot \pi$ o

- ra

L E थ.r an

L. $0.0+$

$4+$ ro ro a $E x$ $n \pm E 0$

a) D.

至

$>0$ + 0

일 L $\frac{1}{\infty}$ i 0 (1) 40 a) u 0 स $=0$

1 in $11>$ a a a $\rightarrow$ a $0+\frac{1}{\omega} \pi$ a) u $\sigma$ व $>05$ a) a c ro $\underline{\omega} a \stackrel{\omega}{\omega} \Omega$ dow $2=1$ a) o 0

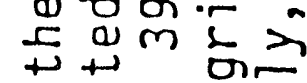
$4+0110$ $0 \frac{0}{a} \rightarrow \frac{0}{x+\pi}$ n a $0 u$ a) $\Rightarrow+\infty$ a) L + a है (4) on a $>$ a $5+L$ त 0 \& $\therefore n^{4}$ त $E \omega$ व -r a $x$ O

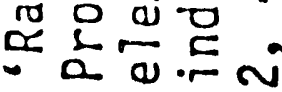
$m$
$\frac{1}{a}$
$\dot{m}$ 


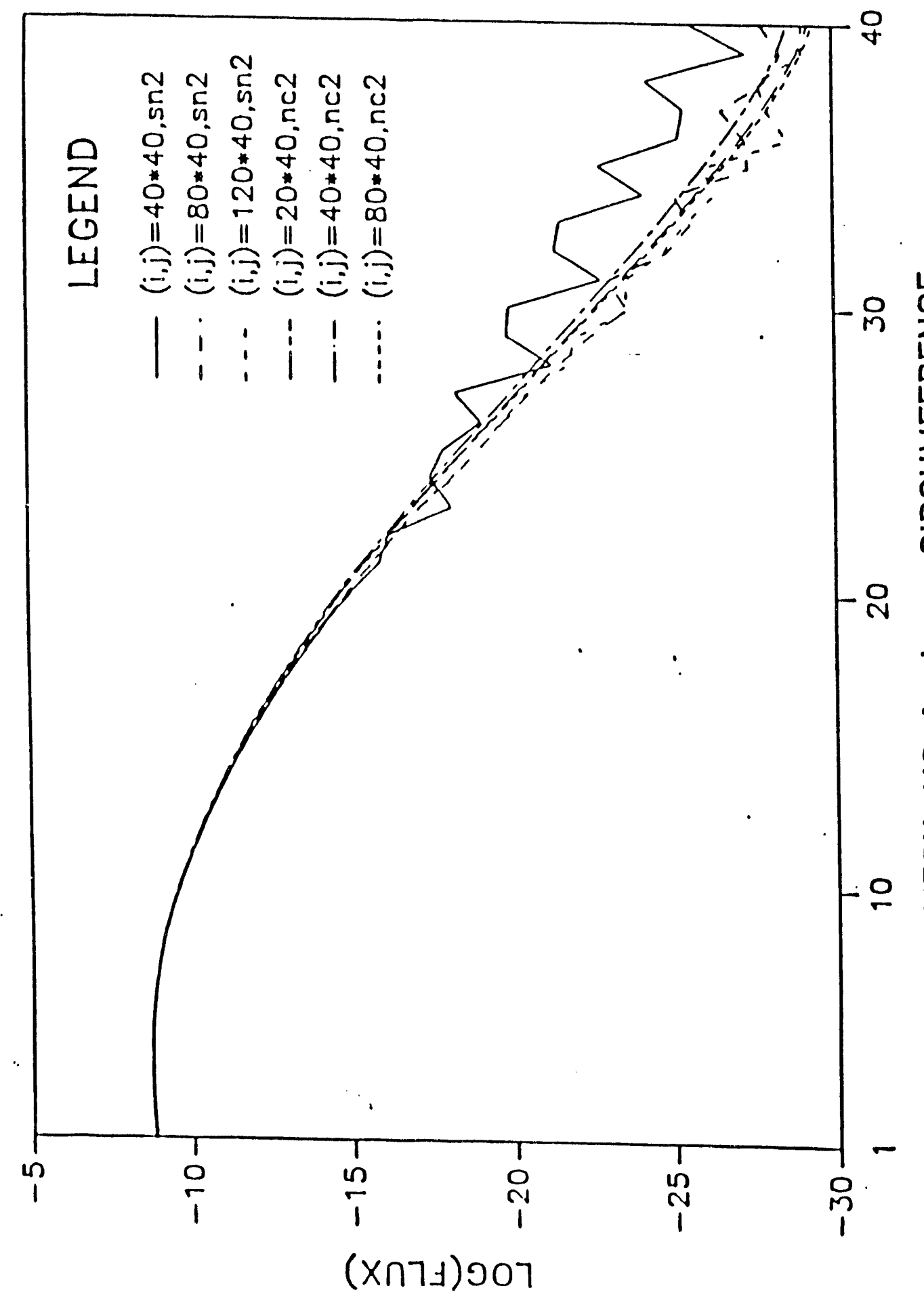

$+$

a)

$2 \sum_{0}^{10} 4$

$4-3$

ก

a a 4 व

$\begin{array}{llll}x & 4 & 4 \\ 4 & 0 & 4\end{array}$

4 \& 0 U

U $\frac{1}{2}+\frac{1}{4} \cdot \frac{1}{2}$

या का व

u $\frac{\pi}{\pi}$ is a

L. 0 as

$\sum$ 도

$3 \quad$ c

() 0 ० 4 ह

0 ortor

○ 5 皮

o $0+5$,

ᄃ 0 य

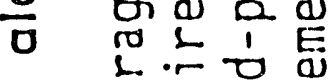

- a O

Z ro as $E$ os

2 a $\frac{\sigma}{2}$

I $E^{+\infty}$

in $4 \cdot \pi+\frac{\pi}{2}$

ᄂ $\rightarrow$ ro

< $2+2$

a a $\uplus$ E a

$+0000$

0 ㅁ․

a $\approx$ ㄴ

Q

C

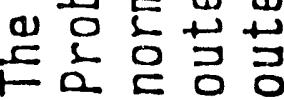

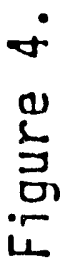



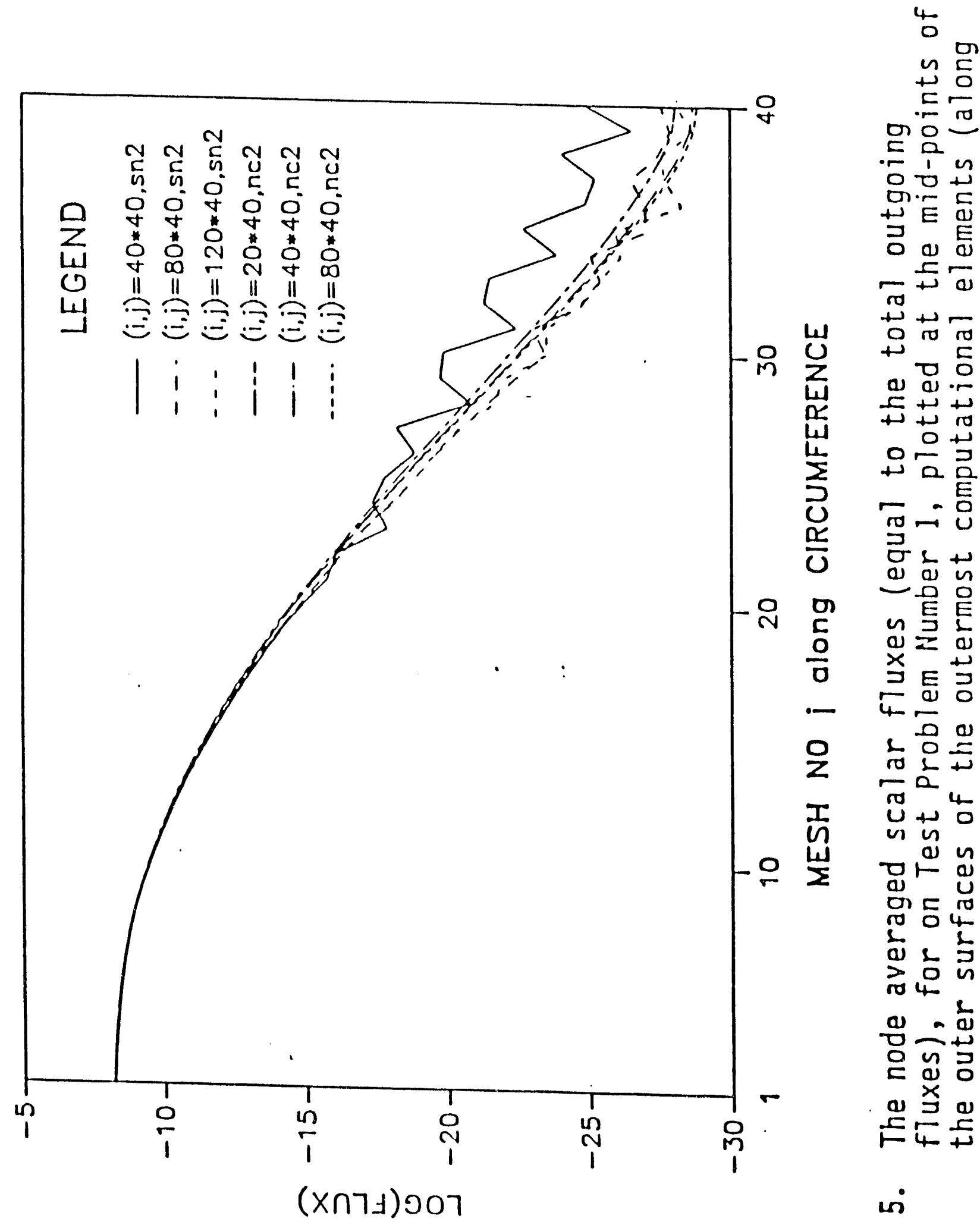

4. 0 ro

U + ए

a) a Q

a $\quad 5+\infty$

i) 0 +

$\sum \quad 0 \sqrt{0}$

() - E

a $\quad \vec{\sigma} \leftarrow U$

( a) a थ

을

대련

$0 \quad x \in \pm$ a

- 4 - 0

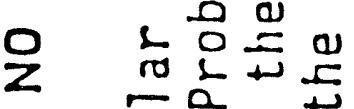

$\frac{\pi}{n} v_{n} 04$

$\longleftarrow$ a $\sim$ a

a $u$

O

L $2 L$

$\rightarrow 25$ a

a $4-2$ a

D $\sim 2 \frac{1}{0}$

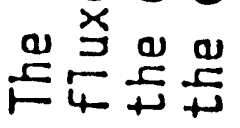

un

$\frac{5}{0}$ 


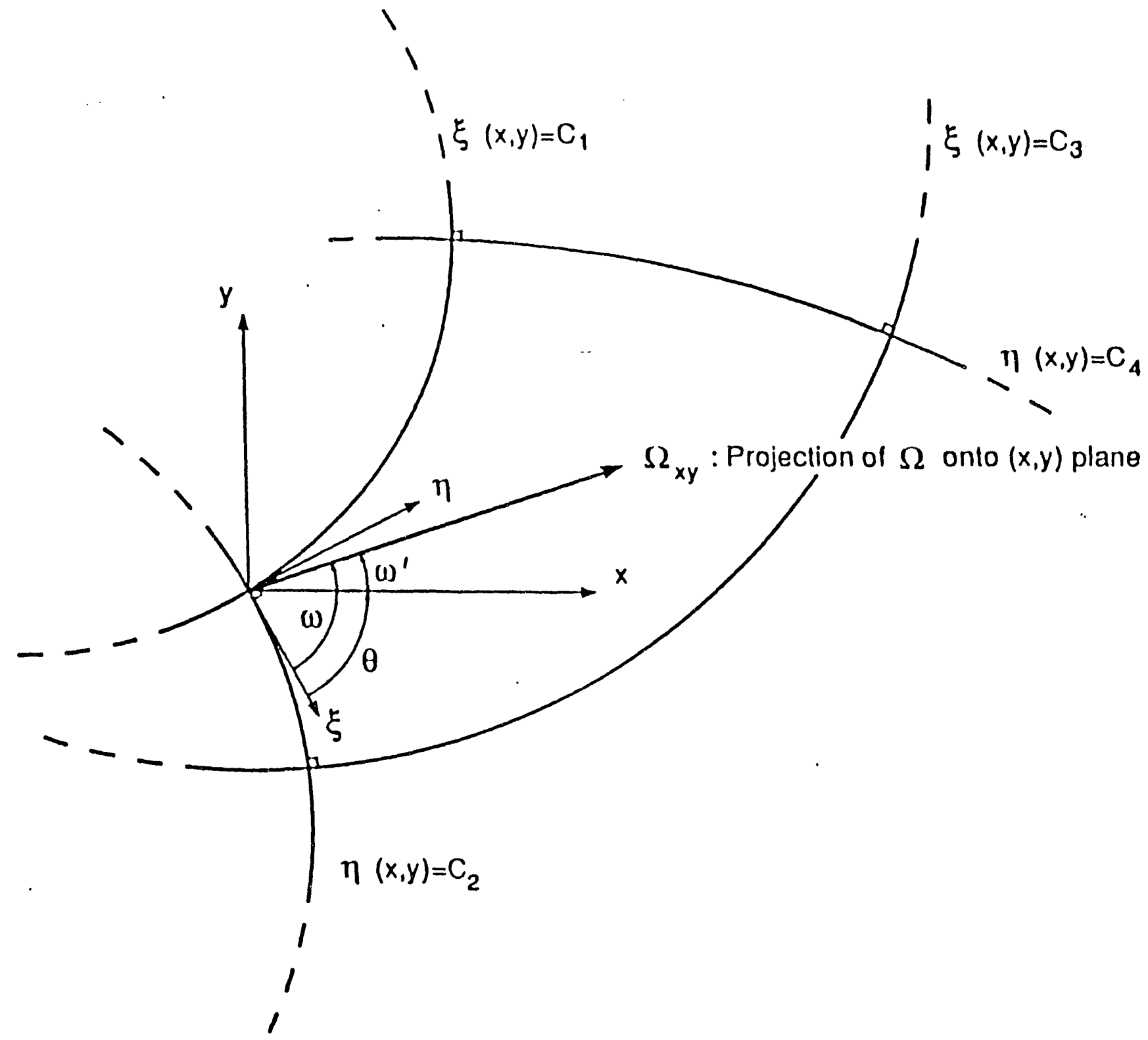

Figure A.1. The general curvilinear courdlinates $(\xi, \eta)$ and the rectangular coordlinates $(x, y)$ in the llane perpendicular to the stralght coordinate $(z)$. The angle $\theta$ is the local rotational angle of the orthogonal curvilinear coordinate system $(\xi, \eta)$ with respect to the fixed rectangular coordinate system $(x, y)$. The normal vector to the $\xi(x, y)=C_{f}$ curve is $\nabla F(x, y)=\xi_{x} \hat{x}+\xi_{y} \hat{y}$ where $F(x, \ddot{y})=$ $\xi(x, y)-C_{1}=0$, and $\tan (0)=\xi_{y} / \xi_{x}$. 
$1-2 * \quad$ U. S. Department of Energy Idaho Field Office 785 DOE Place, MS- 1220

Idaho Falls, ID 83401-1562

Attention: Dr. Joseph H. Nadler

3 U. S. Department of Energy Idaho Field Office 785 DOE Place, MS-1220 Idaho Falls, ID 83401-1562

Attention: Ms. Linda Hallum

4 - 9** J. J. Dorning, Reactor Facility

10 P. A. Allaire, MANE

11 - 12 E. H. Pancake, Clark Hall

$* * *$

SEAS Postaward Administration

13

SEAS Preaward Administration

*1 Original, unbound.

**2 Reproducible copies.

***Cover letter.

JO\#4818:ph 

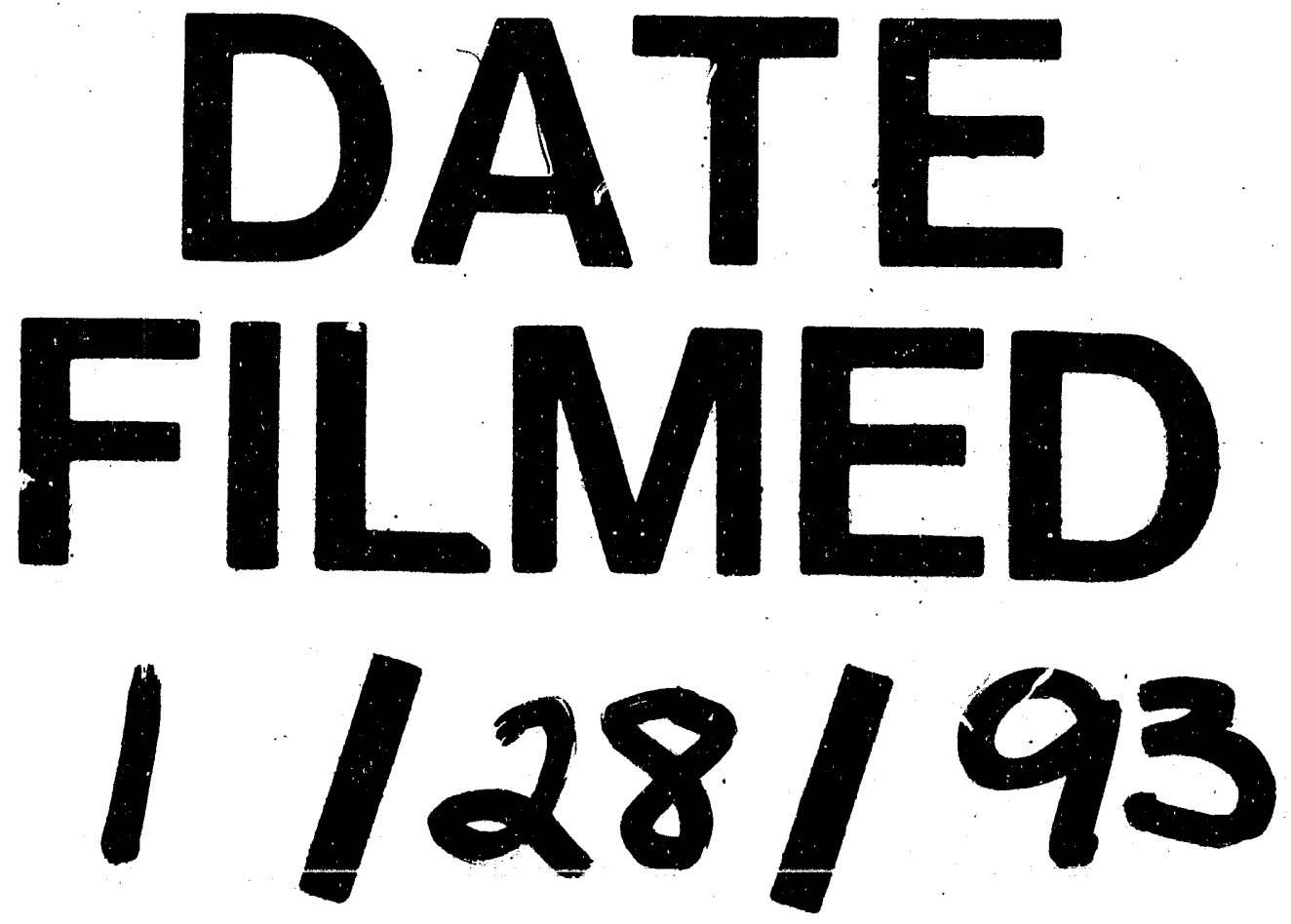
